\title{
Origin of low proton-to-electron temperature ratio in the Earth's plasma sheet
}

E. E. Grigorenko (Space Research Institute of RAS, Moscow, Russia, elenagrigorenko2003@yandex.ru)

E. A. Kronberg, (Max Planck Institute for Solar System Research, Göttingen, Germany, kronbergmps.mpg.de; Ludwig Maximilian University of Munich, Munich, Germany)

P. W. Malv (Max Planck Institute for Solar System Research, Göttingen, Germany, daly@mp mpg.de)

N. Yu. Gaysmina (Finnish Meteorological Institute, Helsinki, Finland, natalia.ganushkina@fmi.fi; University gf Michigan, Ann Arbor, MI, USA)

B. Lavraud (Institut de Recherche en Astrophysique et Planétologie, Université de Toulouse (UPS), France; Eentre National de la Recherche Scientifique, UMR 5277, Toulouse, France, Benoit.La caud@irap.omp.eu)

J.- A. Sauval (Institut de Recherche en Astrophysique et Planétologie, Université de Toulouse (UPS), France Centre National de la Recherche Scientifique, UMR 5277, Toulouse, France, JeanAndre.Sauvaud@irap.omp.eu)

L. M. Zel@vi_Space Research Institute of RAS, Moscow, Russia, lzelenyi@iki.rssi.ru)
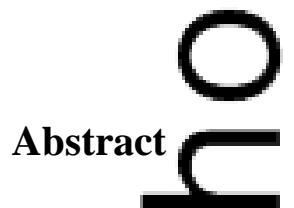

We study the proton-to-electron temperature ratio $\left(T_{\mathrm{p}} / T_{\mathrm{e}}\right)$ in the Plasma Sheet (PS) of the Earth's magnetotant usphg five years of Cluster observations (2001-2005). The PS intervals are searched within a regiondefined with $-19<X \leq-7 \mathrm{R}_{\mathrm{E}}$ and $|Y|<15 \mathrm{R}_{\mathrm{E}}(\mathrm{GSM})$ under the condition $\left|B_{X}\right| \leq 10$ nT. 160 PS crossings are identified. We find an average value of $\left\langle T_{\mathrm{p}} / T_{\mathrm{e}}\right\rangle \sim 6.0$. However, in many

This is the author manuscript accepted for publication and has undergone full peer review but has not been through the copyediting, typesetting, pagination and proofreading process, which may lead to differences between this version and the Version of Record. Please cite this article as doi: 10.1002/2016JA022874 
PS intervals $T_{\mathrm{p}} / T_{\mathrm{e}}$ varies over a wide range from a few units to several tens of units. In 86 PS intervals the $T_{\mathrm{p}} / T_{\mathrm{e}}$ decreases below 3.5. Generally the decreases of $T_{\mathrm{p}} / T_{\mathrm{e}}$ are due to some increase of $T_{\mathrm{e}}$ while $T_{\mathrm{p}}$ either decreases or remains unchanged. In the majority of these intervals the $T_{\mathrm{p}} / T_{\mathrm{e}}$ drops are observed during magnetotail dipolarizations. A superposed epoch analysis applied to these events shows that the minimum value of $T_{\mathrm{p}} / T_{\mathrm{e}}$ is observed after the dipolarization onset during the "turbulent phase" of dipolarization, when a number of transient $B_{Z}$ pulses is reduced, but the value of $B_{Z}$ is stllerge and an intensification of wave activity is observed. The $T_{\mathrm{p}} / T_{\mathrm{e}}$ drops and associated increases or $T_{\mathrm{e}}$ often coincides either with bursts of broad-band electrostatic emissions, which may include eleyclotron harmonics $(\mathrm{ECH})$, or with broad-band electromagnetic emission (EME) in a frequdo from proton plasma frequency $\left(f_{\mathrm{pp}}\right)$ and up to the electron gyrofrequency $\left(f_{c e}\right)$. These findings how that the wave activity developing in the Current Sheet (CS) after dipolarization onset may play a role in the additional electron heating and the associated $T_{\mathrm{p}} / T_{\mathrm{e}}$ decrease.

\section{Key poins}

During trourations transient drops of $T_{\mathrm{p}} / T_{\mathrm{e}}$ below $\sim 3.5$ often coincide with wave bursts in the frequency rans $f_{p p} \leq f \leq f_{\text {ce }}$

Generally the decreases of $T_{\mathrm{p}} / T_{\mathrm{e}}$ are due to some increase of $T_{\mathrm{e}}$ while $T_{\mathrm{p}}$ either decreases or remains unchanged

Electron heming by ECH and EME with $f_{p p} \leq f \leq f_{\text {ce }}$ can be an additional mechanism affecting the $T_{\mathrm{p}} / T_{\mathrm{e}}$ duritg div olarizations.

\section{C.}

\section{Introdulation}

Therm 1 characteristics of the Plasma Sheet (PS) population reflect the energy dissipation processes locating either locally or at a remote location. The important parameter is the protonto-electron temperature ratio $T_{\mathrm{p}} / T_{\mathrm{e}}$, which may influence the Current Sheet (CS) dynamics and 
stability. There are several mechanisms of plasma heating in the Earth's magnetotail and they can operate in different ways for ions and electrons. Ions can be efficiently heated and accelerated in the course of their non-adiabatic interaction with a thin CS or in the vicinity of a magnetic reconnection X-line [e.g. Ashour - Abdalla et al., 1993;1996 and references therein, Hoshino et al., 1998], while electrons can be efficiently heated adiabatically in the course of gradient and curvatur arny in the reconnection pile-up region and by surfatron acceleration at the boundary [e.g. Hoshet al., 2001; 2005; Imada et al., 2007; Fu et al., 2013a], in the contracting and/or coalescing mägnetic islands [e.g. Drake et al., 2006; Oka et al., 2010], in dipolarization events [e.g. Birn l., 2013 and references therein] and in the course of their earthward convection by the conv 1 electric field [Lyons, 1984].

The different efficiencies of the heating mechanisms affect the value of $T_{\mathrm{i}} / T_{\mathrm{e}}$. An important source of $10 \mathrm{t}$ population in the near-Earth tail are bursty bulk flows (BBF), which transport the heated ion from the distant acceleration sources to near Earth [e.g. Angelopoulos et al., 1992; Sergeev et al., 1996; Ohtani et al., 2004; Sharma et al., 2008]. During these periods electrons can experienet adiabatic betatron and/or Fermi accelerations at the associated dipolarization fronts [e.g. Fu et al., 2011, Birn et al., 2013; 2014 and references therein]. These mechanisms are believed to be the major acceleration mechanisms for electrons. However, as it was shown in analytical simulation Zelenyi et al. [1990] and in a recent MHD simulation of magnetotail reconnection, flow burs 5 and dipolarization by Birn et al. [2013] ions, although non-adiabatic experience the similar accereration as adiabatic electrons, at least, in the cases, when the spatial scale of magnetic field gradient 2 ceeds an ion gyroradius and full orbit integration can be applied. This comes from the fact $\mathrm{n}$ the presence of the magnetic field gradient and the dawn-dusk electric field the net energy gain of nonadiabatic ions is obtained at the last part of their orbits because of the difference 
between the duskward and dawnward parts of the ion trajectory. Since this is also the essence of betatron mechanism the last generally should not affect the $T_{\mathrm{i}} / T_{\mathrm{e}}$ ratio.

However, significant variations of $T_{i} / T_{e}$ were reported in many studies using different instruments and different criteria for the plasma region selection. [e.g. Baumjohann et al., 1989; Kaufmatrutert., 2005; Artemyev et al., 2011; Wang et al., 2012; Runov et al., 2015]. Baumjohann et al [198 bv using the AMPTE/IRM data reported that the value of $T_{i} / T_{e}$ ranges between $\sim 5$ and $\sim 10$ with average value $\sim$ 7.0. Similar values of $T_{i} / T_{e}$ were obtained by Kaufmann et al [2005] using the feopil data. For their analysis the authors used observations with $T_{e} \leq 11 \mathrm{MK}$ that, in some cases, may cause an overestimation of $T_{i} / T_{e}$.

Wange al. [2012] used THEMIS observations to study statistically how $T_{\mathrm{i}} / T_{\mathrm{e}}$ ratio changes spatially inthe-magnetosheath and in magnetotail and to identify the processes responsible for these changes. Authors showed that changes in $T_{\mathrm{i}} / T_{\mathrm{e}}$ depend on the initial state of the PS, on the interplanetaryagnetic field (IMF) direction and on AE index. It was demonstrated that during the periods of cover $T_{\mathrm{i}} / T_{\mathrm{e}}$ varies between $\sim 6$ and 10 . This value increases closer to the magnetotail flanks and during the periods of northward IMF. During the period of hot PS and high AE index $T_{\mathrm{i}} / T_{\mathrm{e}}$ decrases and becomes $\sim 2-5$. Authors suggested that the lower values of $T_{\mathrm{i}} / T_{\mathrm{e}}$ can be produced fo nodiabatic heating of electrons. For the near-Earth tail region $\left(X>-10 \mathrm{R}_{\mathrm{E}}\right)$ authors reported strong dawn-dusk asymmetry with very high $T_{\mathrm{i}} / T_{\mathrm{e}}(\sim 15-100)$ observed near the dusk flank andverv low $T_{\mathrm{i}} / T_{\mathrm{e}}(\sim 1)$ registered near the dawn flank. Authors explained this feature by gradient drift of hot ions towards the dusk flank and of hot electrons - towards the dawn flank.

Rano et al. [2015] used THEMIS data to study average thermodynamic properties of the plasma in and around dipolarizing flux bundles (DFB) in the magnetotail at radial distances $5<R<$ 
$25 \mathrm{R}_{\mathrm{E}}$. To select the PS region authors used samples collected within $\left|B_{X}\right|<15 \mathrm{nT}$ for $R<12 \mathrm{R}_{\mathrm{E}}$ and $\left|B_{X}\right|<10 \mathrm{nT}$ for $R>12 \mathrm{R}_{\mathrm{E}}$. They reported that for relatively cold ion populations $\left(T_{\mathrm{i}}<10 \mathrm{keV}\right)$ the average value of $T_{\mathrm{i}} / T_{\mathrm{e}}$ is $\sim 7$, while for the hotter ion population and closer to the Earth (at $R<12$ $\left.\mathrm{R}_{\mathrm{E}}\right) T_{\mathrm{i}} / T_{\mathrm{e}}$ decreases down to $\sim 1.0$. The analysis of 9 events with $T_{\mathrm{i}} / T_{\mathrm{e}} \sim 1$ showed that seven from them were detected near the dawn side of DFB. Authors explained the drop of $T_{\mathrm{i}} / T_{\mathrm{e}}$ by the gradient drifts of io ns and electrons in opposite directions, so that hotter electrons appear near the dawnward edge of DER while hot ions drift duskward.

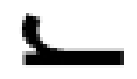

Sereen et al. [2015] using data from six tail seasons of THEMIS observations showed statistically that proton and electron temperature and pressure depend in a different ways on solar wind conditions and substorms. While the proton parameters are well correlated with the solar wind density, verocity and temperature, the behavior of electron temperature and pressure in the PS is mostly co trolled by the substorm-related processes. Authors showed that during the periods of BBFs eled rons experience a stronger heating than protons, so that $T_{\mathrm{i}} / T_{\mathrm{e}}$ can occasionally drop down to $\sim 1$.

Strong variations of proton-to-electron temperature were reported in statistical sudy by Artemyev ot al [2011] based on 4-year of Cluster observations in the near-Earth magnetotail. Authors r portgd that the average value of $T_{p} / T_{e} \sim 3.5$ and it can occasionally decrease below 2.0. Authors ueaproton moments obtained by the Composition and Distribution Function Analyser (CODIFLடRém et al., 2001], which measures proton population in energy range below $40 \mathrm{keV}$. They found stgtistically that the dependence of $T_{\mathrm{p}}$ on $T_{\mathrm{e}}$ can be approximated by the power law function and ortowed that the higher values of $T_{\mathrm{e}}$ are observed during intervals of larger values of the ion and electron bulk velocities. 
Summarizing the previous results one can conclude that the transient processes in the PS associated with the substorm related phenomena (magnetic dipolarizations, bursty bulk flows and so on) may affect electron population in much significant way than the ion one, and cause the decrease of $T_{\mathrm{p}} / T_{\mathrm{e}}$. In the present paper we study in detail the PS dynamics observed during the periods of low $T_{\mathrm{p}} / T_{\mathrm{e}}(\leq 3.5$, the average value reported by Artemyev et al., [2011]) in order to identify the additional mababatic mechanisms which may affect electron and/or proton temperature and cause $T_{\mathrm{p}} / T_{\mathrm{e}}$ dereace For this purpose we study 5 years of Cluster observations in the PS of the near-Earth tail (-19 $\left.<x \leq-7 \mathrm{R}_{\mathrm{E}}\right)$ using the $\left|B_{X}\right| \leq 10 \mathrm{nT}$ as the PS selection criterion. To determine proton temperature used both CODIF data and the observations provided by the 'Research with Adaptive Carte Imaging Detectors' (RAPID) [Wilken et al., 2001] to avoid a possible underestimatio of proton temperature and $T_{\mathrm{p}} / T_{\mathrm{e}}$ value during hot PS periods.

Th Structure of the paper is as follows. In the next section we describe the data selection for

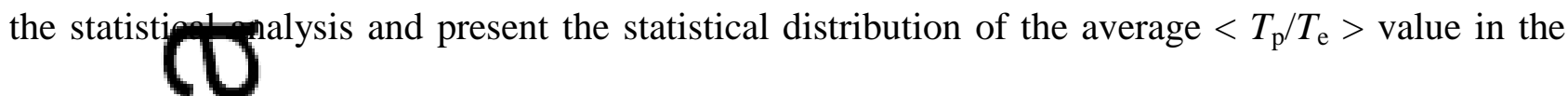
PS. In Section 3 we show one example from our data base in which a $T_{\mathrm{p}} / T_{\mathrm{e}} \sim 2.0$ was observed and discuss the which phase of the PS dynamics the minimum of $T_{\mathrm{p}} / T_{\mathrm{e}}$ is observed. In the last section we summarize ourtresults and discuss the possible mechanisms responsible for the decrease of $T_{\mathrm{p}} / T_{\mathrm{e}}$ in the near-Euthail. In the Appendix we present the list of the PS intervals from our data base, in which $T_{\mathrm{p}} / \mathrm{C}_{\mathrm{e}} .5$ is observed and describe phenomena associated with the $T_{\mathrm{p}} / T_{\mathrm{e}}$ decreases.

2. Dataselection and statistical distribution of $\left\langle T_{p} / T_{e}\right\rangle$

We have analyzed five years of Cluster observations in the near-Earth magnetotail in 20012005. The intervals were selected as the samples when $\left|B_{X}\right| \leq 10 \mathrm{nT}$ within the region with $-19<$ 
$X \leq-7 \mathrm{R}_{\mathrm{E}}$ and $|Y|<15 \mathrm{R}_{\mathrm{E}}$. The magnetic field data are obtained by the fluxgate magnetometer (FGM) [Balogh et al., 2001]. The electron moments are obtained by the Plasma Electron and Current Experiment (PEACE) [Johnstone et al., 1997].

We used observations from Cluster-1 and Cluster-3 spacecraft (hereafter Cl-1 and Cl-3) and found in total 731961 PS samples (4s bins) observed in 160 intervals. The GSM coordinate system was used or oy it and magnetic field data. In Figure 1 we display the scatterplots of the selected PS sampleswin the $(Y Z)$ and $(X Y)$ planes.

For eacn PS sample the values of the proton and electron moments were obtained from the Cluster Sce Archive and interpolated (a linear interpolation was used) to the magnetic field data. It is noting that during periods of strong ion heating the proton temperature and $T_{p} / T_{e}$ value can be underestimated, because the ion energy approaches the upper energy threshold of the CODIF ir strument, and, hence, only the low energy part of the high energy ion population is actually mase in approximately $30 \%$ of the PS crossings the energy corresponding to the peak of proton flux exceeded $20 \mathrm{keV}$. To avoid such bias we performed a visual analyis of the intervals with low values of $T_{\mathrm{p}} / T_{\mathrm{e}}(\leq 4.0)$. For those PS intervals, in which proton energy corresponding to the peak particle flux becomes $\geq 20 \mathrm{keV}$ ( half of the value of the CODIF upper energy threshold), we calculate $T_{p}$ using both CODIF and RAPID observations according testhed described by Daly and Kronberg [2015] and Kronberg et al. [2015]. By combining observations from the two Cluster instruments (CODFI and RAPID) we have found that the minimum trusted value of $T_{p} / T_{e}$ is $\sim 2.0$.

A possi le other bias could come from the entry of low $T_{p} / T_{e}$ plasma populations (of solar wind orint from the flanks during low solar wind Mach number [Lavraud et al., 2009] or northward IMF $B_{Z}$ period $\left[F u\right.$ et al., 2012a].. In such instances a low $T_{p} / T_{e}$ is observed in the 
magnetosheath from overall lower particle heating, and of protons in particular, at the low Mach number bow shock. Subsequent entry of this low $T_{p} / T_{e}$ through the magnetopause was observed [Lavraud et al., 2009]. However, this does not appear to be a possible bias for the low $T_{p} / T_{e}$ events from our data base, since they statistically correspond to more central PS intervals with typical high temperatures, so that the plasma seems heated by local magnetotail processes.

Fig are a presents the statistical 2D distribution of the average values of $\left\langle T_{p} / T_{e}\right\rangle$ in the $(X Y)$ plane within the region of interest. The $\left\langle T_{p} / T_{e}\right\rangle$ were averaged within each $2 \mathrm{R}_{\mathrm{E}} \mathrm{x} 2 \mathrm{R}_{\mathrm{E}}$ bin. To construct this distribution we use only those $(X Y)$ bins in which the number of data samples exceeded hins in which the number of data samples $\leq 10$ are colored white. The majority of the other 0 d bins contains > 100 data samples per bin.

The val of $\left\langle T_{p} / T_{\mathrm{e}}\right\rangle$ averaged over the whole region of study is $\sim 6.0$. The median value of the obser $\mathrm{C}_{\mathrm{p}} / T_{\mathrm{e}}$ is 4.5 . Figure $2 \mathrm{~b}$ displays the $\left\langle T_{\mathrm{p}} / T_{\mathrm{e}}\right\rangle(R)$ profile integrated over all $Y$ locations for a given 18 . One can see that the $\left\langle T_{\mathrm{p}} / T_{\mathrm{e}}\right\rangle$ decreases towards the Earth from $\sim 6.0$ at $R \sim 18 \mathrm{R}_{\mathrm{E}}$ to $\left\langle T_{\mathrm{p}} / T_{\mathrm{e}}\right\rangle \sim 3.0$ at $R \sim 10 \mathrm{R}_{\mathrm{E}}$, which is consistent with the previous results of $T_{\mathrm{i}} / T_{\mathrm{e}}$ spatial behavior obtained bHEMIS observations [Wang et al., 2012; Runov et al., 2015] Unfortunately, magnetotail segments of Cluster orbits during the time period studied do not allow the detailed study of the raal distribution of the $T_{\mathrm{p}} / T_{\mathrm{e}}$ in the PS within the midnight sector $\left(|Y| \leq 5 \mathrm{R}_{\mathrm{E}}\right)$.

Figure shows the $\left\langle T_{p} / T_{\mathrm{e}}\right\rangle(Y)$ profile integrated over all $R$ locations for a given $Y$-bin. Within th $R \sim 10$ to $18 \mathrm{R}_{\mathrm{E}}$ no evident dawn-dusk asymmetry in the $\left\langle T_{p} / T_{e}\right\rangle$ distribution is observear. umere is an increase of $T_{\mathrm{p}} / T_{\mathrm{e}} \sim 20.0$ at $Y \sim-14 \mathrm{R}_{\mathrm{E}}$. Although there are many data samples ( 1500) withi the corresponding $Y$ - bin, this was only a single PS crossing when very hot proton population as observed. 
The absence of dawn-dusk asymmetry in the $\left\langle T_{p} / T_{e}\right\rangle$ distribution obtained from our observations is opposite to the results by Wang et al. [2012], which reported a dawn-dusk asymmetry in the $T_{i} / T_{\mathrm{e}}$ distribution with the smallest values of $T_{i} / T_{e}$ detected at the dawn flank. This discrepancy can be explained by different radial distances between our observations and those of Wang et al. The asymmetry reported by Wang et al. was observed closer to the Earth at $X>-6 \mathrm{R}_{\mathrm{E}}$. The autho suggested that the decrease of the $T_{\mathrm{i}} / T_{\mathrm{e}}$ in the dawn flank and its increase in the dusk flank are accaiated with the gradient drifts of hot ions and electrons towards the dusk and dawn flanks respectively. Near the Earth this effect dominates. However, further downtail other mechanisnm smear out this asymmetry. In the following sections we discuss another possible mechanisi $1 \mathrm{ch}$ can contribute to the decrease of $T_{p} / T_{e}$ in the PS.

Figure $\mathrm{d}$ presents a histogram of the occurrence frequency distribution of the $T_{p} / T_{e}$ observed Tthe PS samples from our data base. It is seen that a wide range of the $T_{p} / T_{e}$ is detected in the PS $10 \leq T_{p} / T_{e}<5.0$. The the lower and upper quartiles are 4.0 and 5.5 respectively. There is also some fraction of the PS samples in which the $T_{p} / T_{e}<3.0$ bserved. An example of such observations is presented in the next section.

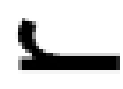

3. Yaigh of the $T_{\mathrm{p}} / T_{\mathrm{e}}$ and associated PS dynamics

It was mentioned above that the $T_{\mathrm{p}} / T_{\mathrm{e}}$ value varies significantly in the PS and can occasionally divp down to 2.0. Figure 3 shows an example of the PS crossing by $\mathrm{Cl}-1$, in which such a phenom non was observed. On 8.10.2001 between 13:20 and 15:50 UT Cluster was located in the maon etoail PS at $[-15.7,9.7,-0.2] \mathrm{R}_{\mathrm{E}}$. During the interval of interest the energy corresponding to the peak electron flux is well below the upper energy threshold of the PEACE 
instrument (see electron Energy-Time spectrogram in Figure3a). However, for protons the energy corresponding to the peak flux approaches to the upper energy threshold of the CODIF instrument (see Energy-Time spectrogram of protons in Figure $3 \mathrm{~b}$ ). Thus, to calculate $T_{\mathrm{p}}$ we used both CODIF and RAPID data.

Fryme time profiles of the $T_{\mathrm{p}}$ and $T_{\mathrm{e}}$ shown in Figure $3 \mathrm{c}$ it is seen that during this event there are erio $\mathrm{s}_{\mathrm{s} \text { wwhen }}$ the temperatures of both species change in a similar way, so that $T_{\mathrm{p}} / T_{\mathrm{e}}$ is roughly cgnstant. Such changes of particle temperature without affecting the $T_{\mathrm{p}} / T_{\mathrm{e}}$ value are most likely cauced the adiabatic mechanisms operating during magnetic dipolarizations and discussed before by Zelenyi et al., [1990], Fu et al., [2011], Birn et al., [2013], Runov et al. [2015]. However, there are also periods when the $T_{\mathrm{p}}$ and $T_{\mathrm{e}}$ either change in an opposite way or their increase (or aecrease) occurs with different speeds. This leads to the observed significant variations in $T_{\mathrm{p}} / T_{\mathrm{e}}$ be $\sim 20.0$ and 2.0 (see Figure $3 \mathrm{~d}$ ). Such strong variations may indicate the importance of additio a possible, nonadiabatic mass-dependent mechanisms of particle heating. We may roughly arect three periods when $T_{\mathrm{p}} / T_{\mathrm{e}}$ becomes $\leq 3.5$, which are marked as "I", "II" and "III" and by verti lines in Figure 3.

Befween 13:20 and 15:50 UT several periods of magnetic dipolarization are observed. The first stron dip larization starts around 14:03 UT with the positive jump $\Delta B_{Z} \sim 23$ nT (see Figure 3f). After Ite onset the $B_{Z}$ field remains larger than it was before the onset and fluctuating during $0.5 \mathrm{~h}$. Five tran rient pulses of the $B_{Z}$ field with the amplitudes $8-16$ nT were observed within $\sim 8$ min after the ginset. At the later phase of the dipolarization event (between 14:11 and 14:19 UT) only one pul of the $B_{Z}$ field was observed (at $\sim 14: 15$ UT). During this later phase, which we will call below as the "turbulent" phase of dipolarization, the $B_{Z}$ field remains still large and experiences 
low-amplitude fluctuations along with the enhancement of wave activity in the frequency range up to electron gyrofrequency $\left(f_{\text {ce }}\right)$ (see Figure $3 \mathrm{~g}, \mathrm{~h}$ ).

The first drop of $T_{\mathrm{p}} / T_{\mathrm{e}}$ (period I) precedes the onset of dipolarization. During this period strong PS flapping is observed: the $B_{X}$ field experiences fast variations between $-15 \mathrm{nT}$ and $+20 \mathrm{nT}$, so that periodically exits to the outer PS. Bursts of wave activity are observed in the electric andonetic field wave spectra measured from $8 \mathrm{~Hz}$ to $4096 \mathrm{~Hz}$ by the Spatio-Temporal Analysis of Field Fluctuations (STAFF) experiment [Cornilleau-Wehrlin et al., 1997] and shown in Figure $3 \mathrm{~g}$. The time profiles of $f_{\text {ce }}$, proton plasma frequency $\left(f_{\mathrm{pp}}\right)$ and the lower hybrid frequency $f_{\mathrm{LH}}=\left(f_{\mathrm{ce}} \cdot f_{\mathrm{c}}\right)^{1 / 2}$ are shown by white, magenta and black lines, respectively. The values of proton and electron temperatures experience variations and $T_{\mathrm{p}} / T_{\mathrm{e}}$ ranges between $\sim 10$ and $\sim 3.0$. We do not discuss the period I in detail, since, because of the fast PS flapping it is difficult to link the $T_{\mathrm{p}} / T_{\mathrm{e}}$

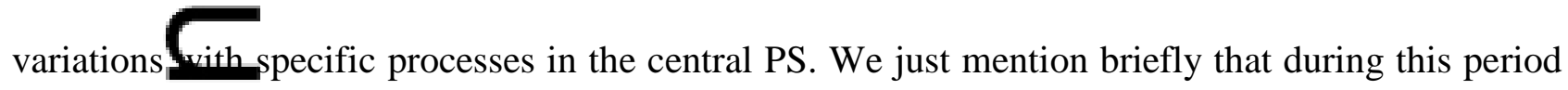
the broad electromagnetic emissions (EMEs) from $0.25 \mathrm{~Hz}$ to several tens of $\mathrm{Hz}\left(\leq f_{\mathrm{pp}}\right)$ are observed Finigher frequencies (up to $f_{\text {ce }}$ ) the electrostatic emission is detected. The power of this emissio in the outer part of the PS (in the region with $\left|B_{X}\right|>10 \mathrm{nT}$ ) and decreases in the central PS,region (not shown). These fluctuations may represent the broad-band electrostatic noise (BEN), whim s often observed in the PSBL and in the outer PS in the course of propagation of field-aligned beams [e.g. Gurnet et al., 1976; Matsumoto et al., 1994]. The study of these phenomenars oeyond the scope of the present paper.

After th dipolarization onset, during period II, the strongest variations of $T_{\mathrm{p}} / T_{\mathrm{e}}$ between 19.0 and 2.0 are served. During this period Cluster was mainly located in the central PS $\left(\left|B_{X}\right| \leq 5 \mathrm{nT}\right)$. A zoom of Cl-1 observations during period II is shown in Figure 4. The absolute minimum of $T_{\mathrm{p}} / T_{\mathrm{e}}$ 
$\sim 2.0$ was observed at 14:12:44 UT, i.e. after the observation of multiple $B_{Z}$ pulses (see Figure $4 \mathrm{e}$ ). Figure $4 \mathrm{~b}$ shows the time profiles of parallel and perpendicular temperatures of protons (black line) and electrons (red lines). Since during this period the energy corresponding to the peak proton flux was well below the upper energy threshold of the CODIF instrument (see proton Energy-Time spectrogram in,Figure 3b) we used the parallel and perpendicular proton temperatures calculated from the QDYF data.

In the heginning of the interval, between 14:10 - 14:10:45 UT, $T_{\mathrm{p}} / T_{\mathrm{e}}$ experiences small variations around $\sim 4.0$ (see Figure 4e). From 14:10:45 UT $T_{\mathrm{p}} / T_{\mathrm{e}}$ starts to decrease. Between 14:10:45 4:11:11:45 UT the decrease of $T_{\mathrm{p}} / T_{\mathrm{e}}$ was due to the significant decrease of $T_{\mathrm{p}}$ and the

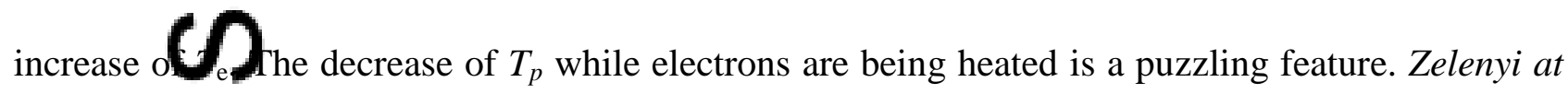
al. [1990] and Birn et al. [2013] showed that the betatron mechanism of electron heating and nonadiabatic on interaction with the CS in the presence of magnetic field gradient provide the similar energy gainfoth plasma components. This condition can be broken if the characteristic spatial scale of magnetic field gradient becomes less than an ion gyroradius $\rho$. The observed anticorrelation between $\Delta T_{p}$ and $\Delta T_{\mathrm{e}}$ may indicate on the existence of the small-scale $\left(\leq \rho_{p}\right)$ magnetic gradients. In such case one can observe only that part of a proton orbit at which particle moves in the direction opposite to the electric field and losses its energy. Another possibility of the $T_{p}$ decrease is the dissipation heir energy due to the interaction with low-frequency waves. Also the observed decrease $\int T_{p}$ can be due to the crossing of different plasma tubes populated by plasma coming from different sources. This puzzling feature deserves a further investigation.

Electrons are heated mainly by betatron mechanism [e.g. Fu et al., 2011; Birn et al., 2013; 2014]. R pmetimes additional mechanisms may contribute to the observed variations of $T_{e}$. In Figure $4 \mathrm{c}$ we present the time profile of electron perpendicular temperature gain measured by 
PEACE instrument at the $i$-th time moment: $\Delta T_{e \perp}(i)=T_{e \perp}(i)-T_{e \perp}(i-1)$ (it is shown by the red line) and the time profile of electron temperature gain expected from the betatron heating: $\Delta T_{e_{-} \text {betatron }}=T_{e \perp}(i-1) \cdot \frac{B(i)}{B(i-1)}-T_{e \perp}(i-1)$ (shown by the black line). One can see that there are time moments, when $\Delta T_{e \perp}$ and $\Delta T_{e_{-} \text {betatron }}$ have opposite signs. At such moments additional mechanis $\$$ m contribute to the observed changes of $\Delta T_{e \perp}$.

m rrgure $4 \mathrm{~d}$ we show a power $\delta B^{2}$ of the magnetic field fluctuations integrated over the $\left[f_{\mathrm{pp}}\right.$, 1

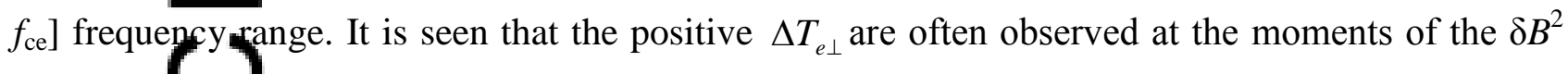
increase. The forrelation coefficient between $\delta B^{2}(t)$ and $\Delta T_{e \perp}(t) C C \sim 0.62$. As it was discussed by Fu et al. anisotropy, which, in turn, can be a source for the whistler wave generation. For a few short intervals period of $T_{\mathrm{p}} / T_{\mathrm{e}}$ decrease we tried to define a link between the changes in electrolabotropy and in the wave spectra. These intervals are marked as "1","2" and "3" in Figure

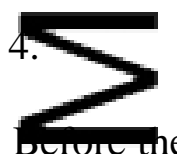

Bervicthe start of interval "1" at 14:11:11 UT the electron distribution was almost isotropic (see Figuce 4b). At 14:11:11 UT the perpendicular anisotropy increases. The moment of $T_{e \perp}$ increa 19 marked in Figure $4 \mathrm{~b}$ by the black arrow. Around this moment $\Delta T_{e \perp}$ and

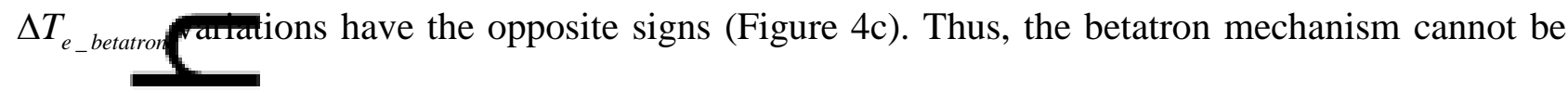
responsiblithe observed increase of electron perpendicular temperature. In Figure 5 we show the energy distri ution of $0^{\circ}, 90^{\circ}$ and $180^{\circ}$ electrons measured at 14:11:11 - 14:11:19 UT along with the time series of 1s elaged spectra of the electric and magnetic field fluctuations observed by STAFF instrument just before and during the two-spin period.. One can see that just $1 \mathrm{~s}$ before the appearance of the 
perpendicular anisotropy the increase of electric field power localized near $f_{c \text { e }}$ is detected. The ECH was observed during $\sim 2 \mathrm{~s}$ and then disappeared. Note that the observation of $\mathrm{ECH}$ after dipolarization onset was previously reported by Zhou et al. [2009]; Zhang and Angelopoulos [2014]. They demonstrated that these fluctuations can energize resonant electrons. The generation of the ECH can be due to the positive slope in $90^{\circ}$ electron distribution observed in the energy range 0.2-1 keV at 14:11:03-14:11:11 UT (not comp) [e.g. Zhou et al., 2009]. We can assume that the ECH can contribute to the perpendicularelectron heating and the increase of the perpendicular anisotropy.

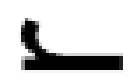

Aprosimately $4 \mathrm{~s}$ after the increase of $T_{e \perp}$ a bulge near $f_{p p}$ appears both in the electric field and magnetigfield power spectra. Figure $4 \mathrm{i}$ shows the direction of the Poynting flux of EME relative to the ambient magnetic field. It is seen that the broad-band EME consists of oblique wave modes in the low frequency range $\left(f \leq f_{\mathrm{pp}} / 2\right)$, which may represent Alfven waves. The waves with $f$ $\sim f_{\mathrm{pp}}$ have Poynting flux directed almost parallel to the ambient magnetic field. During this time Cl-1 $\mathrm{AS}$ bcated mainly in the southern part of the central PS (see the time profile of $B_{X}$ field in Figure so that the wave modes with $f \sim f_{\mathrm{pp}}$ propagated outward from the neutral sheet. Such behavio ropical for whistler modes, which are often observed during magnetic dipolarizations and near a reconnection region [e.g. Petkaki at al. 2006; Le Contel et al., 2009; Viberg et al., 2014, Fu et al., Thus, we may assume that the generation of ECH before the start of interval "1" caused the nerpendicular heating of electrons in a finite energy range and the increase of perpendicular anisotropy. The last, in turn, can be a source for the whistler wave generation with $f \sim$ $f_{\mathrm{pp}}$.

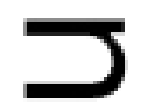

Afte11:19 UT the perpendicular anisotropy in electron distribution decreases. By this time the ECH orsappeared and the magnetic field power corresponding to the bulge near $f_{p \mathrm{p}}$ reduced 
also. The next increase of $T_{e \perp}$ is observed during interval " 2 " at $\sim 14: 11: 30 \mathrm{UT}$ (this moment is marked by the second black arrow in Figure 4b). Similarly to interval "1" the variations of $\Delta T_{e \perp}(t)$ and $\Delta T_{e_{-} \text {betatron }}(t)$ observed during this interval do not correlate. Again the increase of $T_{e \perp}$ is preceded by the observation of ECH at 14:11:25 - 14:11:30 UT (see the corresponding spectra in Figure 5).At 4:11:30 UT a bulge near $f_{\mathrm{pp}}$ appears in the magnetic and electric field power spectra. This bulge copreads to the higher frequency range up to $f_{\text {ce }}$ denoting the generation of the broadband whister EME. This emission disappears rapidly (compare 1s-averaged spectra at 14:11:31 and at 14:11:3 U1 in Figure 5). Comparing the electron distributions measured during the periods "1" and "2" ope the see that in the last period the increase of phase space density $90^{\circ}$ electrons expanded the lower energy range. Similarly to the previous interval we may assume that the ECH contribute to the perpendicular heating of electrons and to the increase of perpendicular anisotropy. The last a source for generation of whistler waves. Possibly the process of electron interaction (Witl the waves had nonlinear character, which manifests in expansion of the EME in higher frequency range up to $f_{\text {ce }}$ and its fast damping due to the absorption of wave energy by resonantercetrons.

Theolute minimum of $T_{\mathrm{p}} / T_{\mathrm{e}} \sim 2.0$ was observed during interval " 3 " at 14:12:44 UT (this moment narke by the blue arrow in Figure 4b). Around this moment both $T_{\mathrm{p}}$ and $T_{\mathrm{e}}$ increased but $T_{\mathrm{e}}$ experic ced the faster increase. The minimum of $T_{p} / T_{\mathrm{e}}$ coincides with the positive variation of $T_{e \perp}$ whil-orresponding variation of $\Delta T_{e_{-} \text {betatron }}$ is negative. Again we may assume that other additionalmed anism contributed to the electron heating.

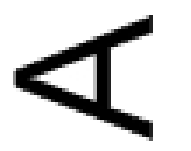


During interval " 3 " the most intense wave emissions were detected. A bulge near $f_{\mathrm{pp}}$ in the spectra of the magnetic and electric field fluctuations was observed permanently from $\sim 14: 12: 20$ UT and until 14:12:40 UT. This bulge can be produced by whistler waves propagating almost along the magnetic field and outward the neutral sheet (see Figure 4f,i). These waves can be generated due to the presence of electron perpendicular anisotropy (Figure 4b). Another possible source for the waves ange related to plasma density and magnetic field gradients [e.g. Le Contel et al., 2009], which can habserved near the leading edge of a high-speed bulk flow. Indeed the $X$-component of proton velocity started to increase in the beginning of interval " 3 " and reached its maximum value $V_{X} \sim 500 \mathrm{k}$ the end of this interval (at 14:13:30 UT, see Figure 4a).

(D)

In Figure 5 we show the electron distribution measured around the $T_{\mathrm{p}} / T_{\mathrm{e}}$ minimum at 14:12:44 -14.13:40 UT and 1s-averaged wave spectra observed during 5s interval preceding the detection fthis distribution. In comparison to the previous intervals the increase of phase space density w $\mathrm{s}$ erved for $90^{\circ}$ electrons in the wider energy range: from a few hundreds of eV and up to $20 \mathrm{ke}$ The STAFF spectra the wave power in the $\left[f_{\mathrm{pp}} f_{\mathrm{ce}}\right]$ range increased at 14:12:40 UT and it was until 14:12:43 UT (see the corresponding spectra in Figure 5). As in the previous periods the EME most likely represents the broad-band whistler EME and consists of wave modes with $f \sim f_{\mathrm{p}}$ propagating almost along the magnetic field outward the CS and the oblique waves with $f_{\mathrm{pp}}<f \leq f_{\text {ce. It }}$ is worth noting that the integral energy density of the EME was of the order of the observed $\Delta \| k T_{\mathrm{e}}$ (not shown). Thus we may assume that the EME can contribute to the electron -

heating obcerved during the first half of interval " 3 " when $\Delta T_{e \perp}$ and $\Delta T_{e_{-} \text {betatron }}$.anticorrelated.

rave energy dissipation just $1 \mathrm{~s}$ before the minimum of $T_{\mathrm{p}} / T_{\mathrm{e}}$ is confirmed by the significant change in the spectral slopes of the electric and magnetic field fluctuations. Indeed the 
spectral indexes $\gamma$ calculated for the electric and magnetic field power in the frequency range between the frequency of the bulge $\left(\sim f_{\mathrm{pp}}\right)$ and $f_{\text {ce }}$ changes from $\gamma_{\mathrm{E}} \sim-1.7$ and $\gamma_{\mathrm{B}} \sim-2.4$ in the beginning of interval " 3 " to $\gamma_{\mathrm{E}} \sim-6.5$ and $\gamma_{\mathrm{B}} \sim-7.0$ just before the $T_{\mathrm{p}} / T_{\mathrm{e}}$ minimum observation. We may suggest that the changes in spectra near $f_{\text {ce }}$ can be caused by the absorption of the wave energy by resonant electrons leading to their perpendicular heating.

In herest of interval " 3 ' the periodic increases and damping of the broad-band whistler EME repeated. At the end of interval "3", when the maximum of the plasma bulk flow was observed gogd correlation between $\Delta T_{e \perp}$ and $\Delta T_{e_{-} \text {betatron }}$ took place. Indeed, the last pronounced increase o $T$ s due to the betatron heating since $\Delta T_{e \perp} \sim \Delta T_{e_{-} \text {betatron }}$.(Figure $4 \mathrm{~b}, \mathrm{c}$ ).

Comin back to the analysis of the PS dynamics between 13:20 nd 15:40 UT one can see that the la decrease of $T_{\mathrm{p}} / T_{\mathrm{e}}$ down to $\leq 4.0$ was observed during the interval III between 14:57 and 15:27 UT 10 igure 3). The $T_{\mathrm{p}} / T_{\mathrm{e}}$ decrease was due to the decrease of $T_{\mathrm{p}}$ and increase of $T_{\mathrm{e}}$ taking place juct hefore the start of the interval. As in period II the exact mechanism responsible for $T_{\mathrm{p}}$ decrease anknown. Before the start of interval III a dipolarization front was observed between 14:50 and 14:56:30 UT. Thus the observed electron heating in the beginning of interval III was produced by betatron mechanism [e.g. Fu et al., 2011]. For protons we may assume that the observed dane of their temperature can be related with the above mentioned effect of a finite spatial sca of the region of magnetic gradient.

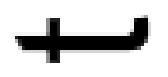

Thet magnetic dipolarization started around 14:57 UT. The $B_{Z}$ field reached its maximum value $\sim 24 \mathrm{nT}$ at 15:05:39 UT. Two $B_{Z}$ pulses were detected within $\sim 9$ min after the onset at 15.20 UT and at 15:05:39 UT. After the last pulse, which corresponds to the absolute 
maximum value of the $B_{Z}$ field reached in this event, the $B_{Z}$ remains large and fluctuates with the smaller amplitude until $~ 15: 14: 30$ UT. This period can be referred to the "turbulent" phase of dipolarization. The fluctuations of $T_{\mathrm{p}} / T_{\mathrm{e}}$ observed during the "turbulent" phase and until the end of the dipolarization (at $\sim 15: 27 \mathrm{UT}$ ) are hardly related to the gradient effects, since no $B_{Z}$ pulses are observed during this time. Within this period the broad-band electrostatic emissions and ECH are detected befth STAFF instrument. In this event the appearance of electron cyclotron fluctuations coincides with the local increase of $T_{e}$ and decrease of $T_{\mathrm{p}} / T_{\mathrm{e}}$ (this moment is shown by black arrow in Figure 3). At this time electron anisotropy (Figure 3e) also decreases to 1.0, suggesting the pitch angle scattonis of electrons.

N

Our observations show that in the course of magnetic dipolarization nonadiabatic mechanismis related to wave-particle interactions may contribute to electron heating and the decrease $\mathrm{d}_{T / T}$. Electrostatic broad-band emissions with ECH and EME with frequencies up to $f_{\text {ce }}$ may resor an ly interact with electrons and cause their energization. In the next session we present the statibaranalysis of the PS dynamics during the periods when the $T_{\mathrm{p}} / T_{\mathrm{e}}$ became $\leq 3.5$ and show that the of such events from our data base are observed after dipolarization onset, when the $B_{Z}$ field is still large and enhancements of in EME and electrostatic fluctuations near $f_{c e}$ are observed.

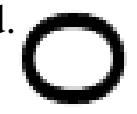

\section{Statisti aranalysis of the $T_{\mathrm{p}} / T_{\mathrm{e}}$ in the PS of the near-Earth tail.}

Wa anatyzed 160 intervals of the PS crossings by Cluster in 2001-2005 time period. In 86 PS intervals ( $54 \%$ ) strong variations and drops of $T_{\mathrm{p}} / T_{\mathrm{e}} \leq 3.5$ were observed. In the majority of these cases 85 intervals) the drops of $T_{\mathrm{p}} / T_{\mathrm{e}}$ were detected during magnetic dipolarizations. The majority of appolarization events in our data base does not represent the isolated earthward 
propagating dipolarization fronts but can be rather referred to the "final" dipolarization events discussed by Nakamura et al. [2009], which can be generated in the near-Earth tail due to the flux pileup. Indeed, the magnetic dipolarizations from our data base represent a prolonged enhancements of the positive $B_{Z}$ field (up to $\sim 10-30 \mathrm{nT}$ ) having a duration from a few tens of minutes and up to a few hours_(see Table 1 in the Appendix). In the majority of cases the onset of these events and the following engal growth of the $B_{Z}$ field are followed by multiple transient pulses of the $B_{Z}$ field, which are really observed during the first $10 \mathrm{~min}$ after the onset and cease at the later phase of the dipolarization. In all these events, except one, the bunches of wave activity in the frequency range up to and higher were detected by the STAFF experiment simultaneously with the $T_{\mathrm{p}} / T_{\mathrm{e}}$ decreases.

In Table 1 of the Appendix we listed these PS intervals containing the $T_{\mathrm{p}} / T_{\mathrm{e}}$ drops below 3.5 and the sultaneous STAFF observations of wave activity. In the majority of these cases the decreases $T_{\mathrm{e}}$ were associated either with the broad-band EME or electrostatic emissions in the frequency range up to $f_{\text {ce }}$, which may heat electrons in the course of their resonant interaction with

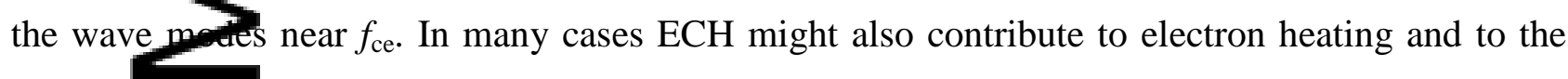
corresponding $T_{\mathrm{p}} / T_{\mathrm{e}}$ decrease.

In oraer to reveal statistically at which phase of magnetic dipolarization and under which conditions the $\Gamma_{\mathrm{p}} / T_{\mathrm{e}}$ decreases we apply a superposed epoch analysis to the PS intervals listed in Table 1 . The epoch analysis was applied to the following parameters: $B_{\mathrm{Z}}, T_{\mathrm{p}} / T_{\mathrm{e}}, T_{\mathrm{p}}, T_{\mathrm{e}}$, the $X$ componemtor proton bulk velocity $\left(V_{X}\right)$, plasma $\beta, A E$ index and the electric and magnetic field wave power $\delta||^{2}(t)$ and $\delta\left(|B|^{2}(t)\right.$ integrated within the frequency range $\left[f_{\mathrm{pp}}, f_{\mathrm{ce}}\right]$. For each event the $B_{Z}$ field normalized to the maximum value of the $B_{Z}$ observed in a given event: $B_{Z} *(t)=$ $B_{Z}(t) / B_{Z}^{\max }$. 
As the epoch time $(t=0)$ we use the dipolarization onset for each event similarly to the previous study by e.g. Fu et al., [2012b]. But in our data base the majority of cases represent rather complicated events with a multiple dipolarization pulses overlapped onto the general prolonged growth of the $B_{Z}$ field (see, for example, the dipolarization event observed between 14:03 and 14:35 UT in Figure 3). To choose the onset (the epoch) time we use one of the following criteria. For the strong dip rarngations, in which the $B_{Z}{ }^{\max }>10 \mathrm{nT}$, the epoch time is chosen as the moment of the first $B_{Z}$ inereace with the amplitude $\Delta B_{Z}=B_{Z i}-B_{Z 0}>5 \mathrm{nT}$, where $B_{Z i}$ is the value of the $B_{Z}$ increase and $B_{\mathrm{Z} 0}$ is the value of the $B_{Z}$ field averaged for $1 \mathrm{~min}$ before the increase. For a few week dipolarizat from our data base with the $B_{\mathrm{Z}}{ }^{\max } \leq 10 \mathrm{nT}$ we choose the onset time as the moment of the firs ive $B_{Z}$ increase with $\Delta B_{Z} \geq 0.5 B_{Z}{ }^{\max }$. In the epoch analysis we also include data up to $15 \mathrm{~min}$ befor the onset.

In some dipolarization events the $B_{Z}$ field can transiently decrease after the onset and increase a vithin ten(s) of seconds denoting the presence of transient dipolarization pulse(s). We consider a yariation of the $B_{Z}$ field observed after the onset as the $B_{Z}$ pulse if $\Delta B_{Z}>5 \mathrm{nT}$ and $\Delta B_{Z} / B_{Z 0}>$ where $\Delta B_{Z}$ is the difference between the peak value of $B_{Z}$ in the pulse and the value $B_{Z 0}$ observed just before the pulse. It is worth noting that in all events from our data base the value of $B_{Z}$ field ooserved after the onset and between the dipolarization pulses does not decrease below its initial veluegistered before the dipolarization onset. Thus, even in the presence of multiple $B_{Z}$ pulses detected after the onset, the average $B_{Z}$ field experiences a gradual growth up to the $B_{Z}{ }^{\max }$, which in some events may last during tens of minutes.

Figured shows the resulting epoch profiles. In order to demonstrate the spread of data used for the extry analysis we present in the corresponding panels of Figure 6 the scatterplots of low and upper quartiles displayed by grey dots. The zero epoch $t=0$ corresponding to dipolarization onset 
in each event is marked by the red vertical line. Along with the epoch profile of the $B_{Z}{ }^{*}(\mathrm{t})$ we show two histograms of the distribution of the average (displayed by the red dotted line) and of the mean (displayed by the sold red line) number of the transient $B_{Z}$ pulses detected within each 10-min bin after the onset of dipolarization events from our data base. It is seen that the maximum number of the $B_{Z}$ pulses is observed within a first $10 \mathrm{~min}$ after the onset. After this time the mean value of the $B_{Z}$ pulses alcy ated for the subsequent time bins is zero and the average value of the $B_{Z}$ pulses is 1.0 within the next three 10-min bins and, then, becomes zero. Thus, following 10 min after the onset a number of transient $B_{Z}$ pulses decreased by $\geq 50 \%$, while the value of $B_{Z}$ still remains large and experts low-amplitude fluctuations. During this period the increase of wave activity in $\left[f_{\mathrm{pp}} f_{\mathrm{ce}}\right]$ rang $\mathrm{i}$ observed. We mark this period by blue horizontal line in Figure 6 and call it as the "turbulent" phase of dipolarization.

Th epoch profile of $\left[T_{\mathrm{p}} / T_{\mathrm{e}}\right](t)$ experiences strong variations around dipolarization onset when the flows are observed. It is worth noting that the $\left[T_{\mathrm{p}} / T_{\mathrm{e}}\right](t)$ enoch profile represents an averaged tendency of how $T_{\mathrm{p}} / T_{\mathrm{e}}$ changes and its single variations (3. drops) are smeared. Plasma $\beta$ experienced strong fluctuations from a few to hundred units around and just after the onset denoting the presence of density gradients. The epoch profiles of $T_{\mathrm{p}}(t)$ and $T_{\mathrm{e}}(t)$ change more or less synchronously that is consistent with the previous results reporting herease of electron temperature with the ion temperature [e.g. Baumjohan et al.; 1989; Arlevev et al. 2011]. However electron temperature increases faster and reaches its maximumearner than proton one. The intense electron heating is observed around and after the onset and during the period of fast bulk flow and the AE maximum This confirms the importance of substorrey ted processes in electron heating reported earlier by Sergeev et al. [2015]. The epoch profile of $T_{\mathrm{e}}(t)$ also shows that the maximum of electron temperature is prolonged in time and the 
"plateau" of large $T_{\mathrm{e}}(t)$ is observed during the first half of the "turbulent" phase of dipolarization, when the intensification of wave activity in high-frequency range (up to $f_{\text {ce }}$ ) are detected.

Our epoch analysis does not show the correlations between $\left[T_{\mathrm{p}} / T_{\mathrm{e}}\right](t)$ and bulk flows. The maximum of $V_{\mathrm{X}}$ coincides with the dipolarization onset as it was observed before in numerous studies [e.g. Ngkamura et al., 2002]. Just after the onset, the positive and negative variations of $V_{\mathrm{X}}$ are obser ed ganoting the registration of the reflected/diversed flows. During this time $\left[T_{\mathrm{p}} / T_{\mathrm{e}}\right](t)$ fluctuates mainly due to $T_{\mathrm{p}}$ fluctuations. While dipolarization proceeds the $V_{X}(t)$ decreases close to zero. This aoes not mean that flow bursts are not observed during this time. The small $V_{\mathrm{X}}$ may denote the perposition of the earthward and reflected flows and/or flow braking. At this later ("turbulen 1 sge of dipolarization the increase of wave activity in $\left[f_{\mathrm{pp}} f_{\text {ce }}\right]$ range is observed and it roughly coincides with the decrease of $\left[T_{\mathrm{p}} / T_{\mathrm{e}}\right](t)$ to the epoch minimum value.

Th IS we may suggest that the minimum of $\left[T_{\mathrm{p}} / T_{\mathrm{e}}\right](t)$ is observed during the "turbulent" phase of distion when the $B_{Z}$ field is still large and the wave activity is enhanced. This suggests that electron interaction with the high frequency electrostatic and EME can be an additional manism providing local electron heating and affecting $T_{\mathrm{p}} / T_{\mathrm{e}}$ during magnetic dipolarizations.

\section{Discussion and Conclusion}

In to identify the PS processes which may affect electron and/or proton temperature and cause the decrease of $T_{\mathrm{p}} / T_{\mathrm{e}}$ we have studied 5 years of Cluster observations in the near-Earth tail at $-19 \times 15 \mathrm{R}_{\mathrm{E}}$ and $|Y|<15 \mathrm{R}_{\mathrm{E}}$ by using the $\left|B_{X}\right| \leq 10 \mathrm{nT}$ as the criterion for PS selection. We have foundand analyzed 160 intervals of PS crossings by Cluster. To avoid underestimation of $T_{\mathrm{p}}$ for the $\mathrm{S}$ intervals, when the energy corresponding to the peak of proton flux exceeded 20 keV.( $30 \%$ of the PS crossings in our data base), we calculate $T_{p}$ using both CODIF and RAPID 
observations [Daly and Kronberg [2015] and Kronberg et al. [2015]. Below we summarize our main results:

1. The value of $T_{p} / T_{\mathrm{e}}$ averaged over the whole region of the PS under study is $\sim 6.0$. This result is more or less in agreement with earlier results obtained from the observations by other space missions [e.g. Baumjohann et al., 1989; Kaufmann et al., 2005]. However, our estimation of the average valuf $T_{\mathrm{p}} / T_{\mathrm{e}}$ is larger than the one obtained in statistical study of Cluster observations by Artemyey [2011]. According to their results the $\left\langle T_{\mathrm{p}} / T_{\mathrm{e}}\right\rangle \sim 3.5$. We explain this discrepancy by $T_{\mathrm{p}}$ calculation using both CODIF and RAPID observations during hot PS intervals while Artemyev et al. [201 only CODIF data.

2. $2 D_{\mathrm{p}} / T_{\mathrm{e}}$ value decreases towards the Earth. from $\sim 6.0$ at $R \sim 18 \mathrm{R}_{\mathrm{E}}$ to $\left\langle T_{\mathrm{p}} / T_{\mathrm{e}}\right\rangle \sim 3.0$ at $R$ $\sim 10 \mathrm{R}_{\mathrm{E}}$, that $\mathrm{i}$ consistent with the previous results of $T_{\mathrm{i}} / T_{\mathrm{e}}$ spatial behavior obtained by THEMIS observatic IS [Wang et al., 2012; Runov et al., 2015]. Within the radial distances -9 to $-19 \mathrm{R}_{\mathrm{E}}$ no evident day ask asymetry in the $\left\langle T_{p} / T_{e}\right\rangle$ distribution is observed.

3. Within a single PS interval the value of $T_{p} / T_{e}$ may fluctuate in a wide range from a few units to several tens of units. According to our observations the minimum trusted value (from instrument limitations of Cluster) of $T_{p} / T_{e}$ in the PS is $\sim 2.0$. The decreases of $T_{p} / T_{e}$ below 3.5 (the average estumation obtained by Artemyev et al. [2011]) were detected in 86 PS intervals from our data base. In majority of these cases (in 85 intervals) the drops of $T_{\mathrm{p}} / T_{\mathrm{e}}$ were observed during magnetic ipolarizations.

4. Ime superposed epoch analysis applied to the dipolarization events in which decreases of $T_{\mathrm{p}} / T_{\mathrm{e}} \leq 3.5$ we observed in the PS shows that the $T_{\mathrm{p}} / T_{\mathrm{e}}$ experiences strong variations around the dipolarizuro onset and decreases after the onset, during the "turbulent" phase of dipolarization, when the $B_{Z}$ field in the CS is still large. The time interval of $T_{\mathrm{p}} / T_{\mathrm{e}}$ drop below 3.5 coincides with 
the enhancement of electric and magnetic field wave power in $\left[f_{\mathrm{pp}}, f_{\mathrm{ce}}\right]$ frequency range. This denotes that the high frequency electrostatic and EME may play some role in electron heating and $T_{\mathrm{p}} / T_{\mathrm{e}}$ decrease during magnetic dipolarizations.

Before discussing the results we would like to note that one should be very careful with the evaluation of the $T_{\mathrm{p}} / T_{\mathrm{e}}$ value. The upper energy threshold (40 keV) of the CODIF instrument may result in cergstimation of $T_{\mathrm{p}}$ and $T_{\mathrm{p}} / T_{\mathrm{e}}$ values during active PS periods, when bursty bulk flows, dipolarization fronts and other perturbations are observed [e.g. Angelopoulos et al. 1992, Runov et al., 2009]. For such periods the use of only CODIF observations may give values as low as $T_{p} / T_{\mathrm{e}} \leq$ 1.0. The vexamination of proton energy-time spectrograms showed that such low values are indeed modye to the unusually strong proton heating (with limitations owing to the CODIF upper energy treshold). Using RAPID measurements along with the CODIF data allowed us to obtain the eliable minimum value of the $T_{\mathrm{p}} / T_{\mathrm{e}} \sim 2.0$ for the PS intervals from our data base.

In 10 all intervals from our database the decreases of $T_{\mathrm{p}} / T_{\mathrm{e}}$ below 3.5 were detected in the PS during magnetic dipolarizations. The magnetic dipolarizations may represent earthward propagating ints, which are generated by a downtail reconnection [e.g. Sitnov et al., 2009; Fu et $a l ., 2013]$. Or they can be related with the CS reduction/disruption either due to the development of CS instabintues [Lui 2004 and references therein] or in response to fast flow braking [e.g. Sergeev et al., 2012 andeferences therein]. Nakamura et al. [2009] classified such events as the "final" dipolarizalion due to the flux pileup. The evolution of these events in time and space can be complicattuand include multiple transient dipolarizations around and after the onset.

Many bservations and simulations reported significant electron heating at and behind dipolarizero fronts by betatron and Fermi mechanisms [e.g. Fu et al., 2011; Brin et al., 2013; Birn et al., 2014 and references therein]. However, as it was shown in theoretical paper by Zelenyi et al. 
[1990] and in simulations by Birn et al. [2013], ions, although nonadiabatic, undergo the similar energization as electrons. Thus generally these major mechanisms of plasma heating hardly affect the $T_{p} / T_{e}$.

Artemyev et al. [2011] and Wang et al. [2012] proved statistically that the thermal characteristics of electron population and $T_{\mathrm{p}} / T_{\mathrm{e}}$ value depend on the background state of the PS and generally $1 \mathrm{C}$ igher and $T_{\mathrm{p}} / T_{\mathrm{e}}$ is lower in hot PS. Artemyev et al. [2011] also demonstrated that $T_{\mathrm{e}}$ increases during the period of high-speed plasma flows. Sergeev et al. [2015] showed a preferential heating of electrons and drop of $T_{\mathrm{i}} / T_{\mathrm{e}}$ during the periods of BBFs.

Oufoch superposition analysis showed that $T_{\mathrm{p}}(t)$ and $T_{\mathrm{e}}(t)$ change more or less synchrono $1 \mathrm{n}$ the course of magnetic dipolarization which is consistent with results by Artemyev et al. [2011]. However at the onset and just after the onset the $T_{\mathrm{e}}(t)$ increases more rapidly than $T_{\mathrm{p}}(t)$. The Intense electron heating coincides with the increase of the bulk $V_{\mathrm{X}}$ velocity at the dipolarization made by Sergeev et al. [2015] that the thermal characteristics of electrons are affected stronger than ion ones diving substorm-related processes.

However the minimum of the epoch profile of $\left[T_{\mathrm{p}} / T_{\mathrm{e}}\right](t)$ is observed after the dipolarization onset durifing "turbulent phase" of dipolarization when the $B_{Z}$ is still large and the intensification of wave a y in $\left[f_{p p}, f_{c e}\right]$ frequency range is observed. First of all we would like to check how instantane us values of $T_{\mathrm{p}}$ and $T_{\mathrm{e}}$ changes near the absolute minimum of $T_{\mathrm{p}} / T_{\mathrm{e}}$ observed in the PS intervals $1 / 1 \mathrm{~m} / T_{\mathrm{e}} . \leq 3.5$. In Figure 7 we present a scatterplot of $\Delta T_{\mathrm{e}}(i)=T_{\mathrm{e}}(i)-T_{\mathrm{e}}(i-1)$ versus the corresponding $(T p(i)$ calculated for each PS interval from our data base at the $i$-th time moment at which th solute minimum of $T_{\mathrm{p}} / T_{\mathrm{e}}$ was observed. It is seen that in majority of cases form our data base the minimum of $T_{\mathrm{p}} / T_{\mathrm{e}}$ is reached due to the $T_{\mathrm{e}}$ increase and $T_{\mathrm{p}}$ decrease and generally the 
decrease of $T_{\mathrm{p}}$ is larger than the corresponding increase of $T_{\mathrm{e}}$. Such anticorrelation in $T_{\mathrm{p}}$ and $T_{\mathrm{e}}$ changes is a puzzling feature. As it was mentioned before, under the presence of magnetic field gradient and the dawn-dusk electric field the adiabatic electrons, in the course of their betatron heating, and non-adiabatic ions, in the course of their nonadiabatic motion in the CS, obtain the similar energy gain. The opposite signs of temperature changes observed for protons and electrons can be rel ted some kinetic effects of proton dynamics in the CS. Birn et al [2014] mentioned the

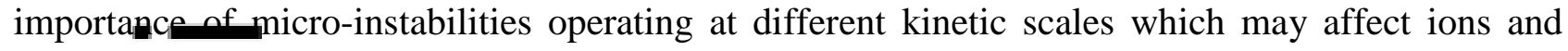
electrons at anferent ways. Another possibility is the presence of small-scale (less than proton gyroradius gnetic gradients. In such case the full orbit integration is not applied and an observer can detect passing only that part of their orbits, at which they lose energy due to the motion antiparallel to he electric field. Also the transient decreases of $T_{p}$ can be due to the spacecraft crossings or different plasma tubes connected with different sources. The exact mechanism responsibl the transient $T_{p}$ decreases in the course of magnetic dipolarization is an open question which deserves farther investigation.

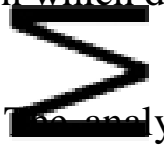

ysis of STAFF observations of the electric and magnetic flied fluctuations in the frequency range from $8 \mathrm{~Hz}$ to $4096 \mathrm{~Hz}$ permitted the identification of several wave modes, which generation wides with the $T_{p} / T_{e}$ decreases. These modes are listed in Table 1 for each PS interval from our data base when the drops of $T_{p} / T_{e} \leq 3.5$ were observed (see the Appendix).

III tII Thajority of cases the decreases of $T_{p} / T_{e}$ coincide either with the observations of broadbandEME or broadband electrostatic emissions. The last includes ECH in $\sim 50 \%$ of events. The observations of these wave modes during magnetic dipolarizations were reported in previous studies E.o Contel et al., 2009; Deng et al., 2010, Hwang et al., 2011, Zhou et al. 2009; Fu et 
al., 2014; Zhang and Angelopoulos, 2014]. The broad-band EME comprise from the whistler waves in the frequency range $f \leq f_{\mathrm{pp}}$, which propagate outward from the CS almost parallel to the magnetic field and oblique whistler waves in the higher frequency range $f_{\mathrm{pp}}<f \leq f_{\text {ce. }}$. For higher frequency modes we observed an increase of the spectral index, $\left|\alpha_{\mathrm{E}}\right|$, denoting the significant reduction of the

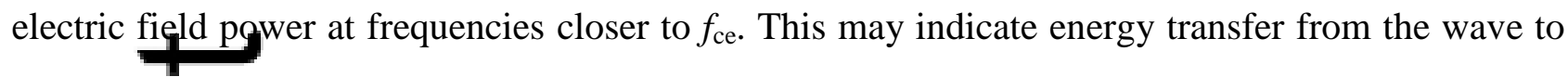
resonant e ectryns.

Khetyaintsev et al. [2011] discussed the processes of wave-particle interaction in the flow braking region and showed that whistler-mode waves can efficiently scatter electrons in pitchangles and thus, increase the efficiency of betatron acceleration for some part of electron distribution. They also demonstrated that the particle interaction with whistler modes limits the electron anisotropy caused by the betatron acceleration at lower energies, so that the resulting distributio has limited anisotropy below $\sim 2 \mathrm{keV}$, and is more anisotropic at higher energies. Our analysis of edron pitch angle distributions presented in Section 3 showed similar features for the periods $T_{p} / T_{e}$ drops associated with the ECH and broad-band EME. We observe electron pitch-an the energy range below $\sim 500 \mathrm{eV}$ and perpendicular anisotropy at energies from $\sim 50 \mathrm{eV}$ and up to several $\mathrm{keV}$. Also, a pronounced flat-top electron distribution feature was observed fores pitch angle electrons in the energy range $0.1-3 \mathrm{keV}$, suggesting perpendicular electron heating.

Thenostibility of electron energization and scattering by ECH was discussed before by, e.g. Farrell et al. 2003] and Zhou et al. [2009]. Our analyses also showed that in many events the observation broadband electrostatic fluctuations and electron cyclotron waves coincides with a 
local increase of $T_{e}$ and a decrease of $T_{\mathrm{p}} / T_{\mathrm{e}}$. At such times the electron anisotropy $T_{e \|} / T_{e \perp}$ also decreases to 1.0, suggesting efficient electron pitch angle scatterings.

However it is rather difficult to reveal cause-and-effect relation between the generation of waves, kinetic effects in particle distributions and particle energization. As it was discussed by $F u$ et al. [201\% 2012a; 2014], the perpendicular anisotropy of electron distributions appeared due to the betatrcating can be a source for wave generation. However the generated waves may, in turn, inteact with the resonant electrons, so that at the later stage this process can become nonlinear. (t 13 worth noting also that the particle anisotropy is not the only source of whistler waves. They ge generated also due to the presence of plasma density gradients and magnetic field gradientc_e.g. Le Contel et al., 2009 and references therein] which are formed in the course of dipolarizations. Propagating through the background plasma these waves can interact with the ambient elewon population and cause its heating.

\section{(T)}

Our analysis presented in Section 3 showed that during some intervals of $T_{\mathrm{p}} / T_{\mathrm{e}}$ decrease the periodical enthement and damping of ECH and EME emissions in frequency range $\left[f_{\mathrm{pp}}, f_{\mathrm{ce}}\right]$ are observed in spite of the almost permanent presence of the perpendicular anisotropy in electron distributionso it was shown that the increases of wave power in $\left[f_{\mathrm{pp}}, f_{\text {ce }}\right]$ range more or less correlate ith local increases of electron temperature $\Delta T_{\mathrm{e}}$ (see Figure $4 \mathrm{c}, \mathrm{d}$ ). The strong increase of spectra index $\gamma$ observed in the spectra of electric and magnetic field fluctuations in frequency range [ $f_{\mathrm{pp}}$ ange just before the minimum of $T_{\mathrm{p}} / T_{\mathrm{e}}$ (see Figure 5) confirms the possibility of energy exchenge between the waves and resonant electrons.

Gi ure 8 we present the scatterplots of $T_{\mathrm{e}}\left(\right.$ and $T_{\mathrm{p}} / T_{\mathrm{e}}$ ) versus the power of magnetic and electric field fluctuations $\delta B^{2}, \delta E^{2}$ integrated in $\left[f_{\mathrm{pp}} f_{\mathrm{ce}}\right]$ frequency range for all time moments within 
the PS intervals in which $T_{\mathrm{p}} / T_{\mathrm{e}} \leq 3.5$ was observed. Although data points are rather scattered there is a tendency to observe larger $T_{\mathrm{e}}$ with the increase of the fluctuations power. This tendency is more clearly observed for the electric field fluctuations, which may indicate on the role of high-frequency electrostatic emission in the electron heating. For $T_{\mathrm{p}} / T_{\mathrm{e}}$ there is a tendency to observe lower values for the higher power of fluctuations. Again this tendency is more clearly observed for the electric field fluct ations.

Summarizing our results we may conclude that the processes of electron interactions with the ECH and EME emissions in $\left[f_{\mathrm{pp}} f_{\mathrm{ce}}\right]$ frequency range may play some role and provide some heating of trons additionally to the major energization mechanisms like betatron and/or Fermi acceleratid hich mechanisms are responsible for the simultaneously observed $T_{p}$ decrease during the peri ds of low $T_{\mathrm{p}} / T_{\mathrm{e}}$ is still an open question and requires farther studies. It is worth noting tha che changes in $T_{p} / T_{e}$ and the related changes in particle velocity distribution functions may, in tupfect the CS dynamics and the development of plasma instabilities. These problems deserve special theoretical and modeling studies, which may shed new lights on CS dynamics during magnetic dipolarizations.

\section{Appencix}

In 1 we list all the PS intervals when the $T_{p} / T_{e} \leq 3.5$ was observed. In the majority of these case the decrease in $T_{p} / T_{e}$ was registered during magnetic dipolarizations. The analysis of STAFF ouservations of the electric and magnetic flied fluctuations in the frequency range from 8 $\mathrm{Hz}$ to $4096 \mathrm{~Hz}$ permitted the identification of wave modes, observed in the PS simultaneously with the $T_{p} / T$ ecc ease below 3.5. These modes are also listed in the Table 1. 
Table 1. A list of the PS intervals when the $T_{p} / T_{e} \leq 3.5$ is observed

\begin{tabular}{|c|c|c|}
\hline Time Interval & $\begin{array}{l}\text { Magne } \\
\text { tic } \\
\text { dipolarization }\end{array}$ & Wave activity \\
\hline$-17: 00 \mathrm{UT}$ & yes & broadband EME $\left(f \leq f_{\mathrm{ce}}\right)$, whistler waves \\
\hline 26.07.01.17:17 - 17:59 UT & yes & broadband EME $\left(f \leq f_{\text {ce }}\right)$, whistler waves \\
\hline $\begin{array}{r}26.07 .01 . \\
.\end{array}$ & yes & $\begin{array}{l}\text { broadband electrostatic fluctuations }\left(f \leq f_{\text {ce }}\right) \text {, } \\
\text { electron cyclotron waves }\end{array}$ \\
\hline 27.07.01. & yes & $\begin{array}{l}\text { broadband electrostatic fluctuations }\left(f \leq f_{\mathrm{ce}}\right) \text {, } \\
\text { whistler waves }\left(f \leq f_{\mathrm{pp}}\right)\end{array}$ \\
\hline - 10:37 UT & yes & $\begin{array}{l}\text { broadband electrostatic fluctuations }\left(f \leq f_{\mathrm{ce}}\right) \text {, } \\
\text { electron cyclotron waves }\end{array}$ \\
\hline $21: 37 \mathrm{UT}$ & yes & $\begin{array}{l}\text { broadband EME }\left(f \leq f_{\text {ce }}\right) \text {, electron cyclotron } \\
\text { waves, whistler waves }\end{array}$ \\
\hline 08.08 .01 & yes & $\begin{array}{l}\text { broadband electrostatic fluctuations }\left(f \leq f_{\text {ce }}\right) \text {, } \\
\text { electron cyclotron waves }\end{array}$ \\
\hline $05-19: 00 \mathrm{UT}$ & yes & broadband EME $\left(f \leq f_{\text {ce }}\right)$, whistler waves \\
\hline 15.08.01. 1:47-02:15 UT & yes & broadband electrostatic fluctuations $\left(f \leq f_{\mathrm{ce}}\right)$ \\
\hline 15.08.01. 05:33-07:40 UT & yes & broadband electrostatic fluctuations $\left(f \leq f_{\mathrm{ce}}\right)$ \\
\hline 15.08.01. 810s-10:11 UT & yes & broadband EME $\left(f \leq f_{\text {ce }}\right)$, electron cyclotron waves \\
\hline 22.08.01. No-10:26 UT & yes & broadband electrostatic fluctuations $\left(f \leq f_{\text {ce }}\right)$ \\
\hline$-05: 50 \mathrm{UT}$ & yes & $\begin{array}{l}\text { broadband EME }\left(f \leq f_{\text {ce }}\right) \text {, whistler waves, electron } \\
\text { cyclotron waves }\end{array}$ \\
\hline$-12: 36 \mathrm{UT}$ & yes & broadband electrostatic fluctuations $\left(f \leq f_{\mathrm{ce}}\right)$ \\
\hline $07.09 .01 .21: 56-22: 10 \mathrm{UT}$ & yes & broadband electrostatic and EME $\left(f \leq f_{\text {ce }}\right)$ \\
\hline 12.09.01. $3: 15-13: 45$ UT & yes & broadband electrostatic and EME $\left(f \leq f_{\mathrm{ce}}\right)$ \\
\hline 15.09.01. 00:40-01:30 UT & yes & broadband electrostatic and EME $\left(f \leq f_{\mathrm{ce}}\right)$ \\
\hline 17.09.01. 8:14-10:07 UT & yes & $\begin{array}{l}\text { broadband electrostatic }\left(f \leq f_{\text {ce }}\right) \text { and electron } \\
\text { cyclotron waves }\end{array}$ \\
\hline - 11:00 UT & yes & $\begin{array}{l}\text { broadband EME }\left(f \leq f_{\text {ce }}\right) \text {, whistler waves, electron } \\
\text { cyclotron waves }\end{array}$ \\
\hline $4-13: 41 \mathrm{UT}$ & yes & $\begin{array}{l}\text { broadband EME }\left(f \leq f_{\text {ce }}\right) \text {, whistler waves, electron } \\
\text { cyclotron waves }\end{array}$ \\
\hline $08.10 .01 . \mathrm{T}$ & yes & broadband EME $\left(f \leq f_{\mathrm{ce}}\right)$, whistler waves \\
\hline 08.10.01. & yes & $\begin{array}{l}\text { broadband electrostatic fluctuations, electron } \\
\text { cyclotron waves }\end{array}$ \\
\hline$-05: 30 \mathrm{UT}$ & yes & $\begin{array}{l}\text { broadband electrostatic and EME }\left(f \leq f_{\mathrm{ce}}\right) \text {, whistler } \\
\text { waves }\end{array}$ \\
\hline 20.10.01. 10:47 - 13:05 UT & yes & broadband electrostatic $\left(f \leq f_{\text {ce }}\right)$ and electron \\
\hline
\end{tabular}




\begin{tabular}{|c|c|c|}
\hline & & cyclotron waves \\
\hline 21.07.02. 03:40 - 05:00 UT & yes & broadband electrostatic waves $\left(f \leq f_{\text {ce }}\right)$ \\
\hline 21.07.02. 09:42-13:03 UT & yes & $\begin{array}{l}\text { broadband EME }\left(f \leq f_{\text {ce }}\right) \text {, whistler waves, electron } \\
\text { cyclotron waves }\end{array}$ \\
\hline 21.07.02. 13:48-14:51 UT & yes & $\begin{array}{l}\text { broadband electrostatic waves }\left(f \leq f_{\text {ce }}\right) \text {, whistler } \\
\text { waves, electron cyclotron waves }\end{array}$ \\
\hline 25.07.02.22:26-23:58 UT & yes & $\begin{array}{l}\text { broadband electrostatic }\left(f \leq f_{\text {ce }}\right) \text { and electron } \\
\text { cyclotron waves }\end{array}$ \\
\hline - 08:00 UT & yes & $\begin{array}{l}\text { broadband electrostatic }\left(f \leq f_{\text {ce }}\right) \text { and electron } \\
\text { cyclotron waves }\end{array}$ \\
\hline - 19:05 UT & yes & broadband electrostatic waves $\left(f \leq f_{\text {ce }}\right)$ \\
\hline$=02: 22 \mathrm{UT}$ & yes & broadband electrostatic waves $\left(f \leq f_{\text {ce }}\right)$ \\
\hline 14.10.0Z. T2.20 - 14:10 UT & yes & $\begin{array}{l}\text { broadband electrostatic waves }\left(f \leq f_{\text {ce }}\right) \text {, electron } \\
\text { cyclotron waves }\end{array}$ \\
\hline 14.10 .02 . & yes & $\begin{array}{l}\text { broadband electrostatic waves }\left(f \leq f_{\text {ce }}\right) \text {, electron } \\
\text { cyclotron waves }\end{array}$ \\
\hline$-08: 22 \mathrm{UT}$ & yes & broadband electrostatic waves $\left(f \leq f_{\mathrm{ce}}\right)$ \\
\hline 20.07 .03 .60 & yes & $\begin{array}{l}\text { broadband EME }\left(f \leq f_{\text {ce }}\right) \text {, whistler waves, electron } \\
\text { cyclotron waves }\end{array}$ \\
\hline 20.07.03. 05:04-05:37 UT & yes & broadband electrostatic waves $\left(f \leq f_{\mathrm{ce}}\right)$ \\
\hline 20.07.03. 11:00 UT & yes & broadband electrostatic waves $\left(f \leq f_{\mathrm{ce}}\right)$ \\
\hline 24.07.03.22:40 UT & yes & broadband electrostatic waves $\left(f \leq f_{\text {ce }}\right)$ \\
\hline$-24: 00 \mathrm{UT}$ & yes & broadband EME $\left(f \leq f_{\text {ce }}\right)$, whistler waves \\
\hline$-08: 39 \mathrm{UT}$ & yes & $\begin{array}{l}\text { broadband EME }\left(f \leq f_{\mathrm{ce}}\right) \text {, whistler waves, electron } \\
\text { cyclotron waves }\end{array}$ \\
\hline 15:24 UT & yes & $\begin{array}{l}\text { broadband electrostatic waves }\left(f \leq f_{\text {ce }}\right) \text {, electron } \\
\text { cyclotron waves }\end{array}$ \\
\hline $55-15: 11 \mathrm{UT}$ & yes & broadband electrostatic waves $\left(f \leq f_{\mathrm{ce}}\right)$ \\
\hline $33-21: 31$ UT & yes & $\begin{array}{l}\text { broadband electrostatic waves }\left(f \leq f_{\text {ce }}\right) \text {, electron } \\
\text { cyclotron waves }\end{array}$ \\
\hline - 02:06 UT & yes & $\begin{array}{l}\text { broadband electrostatic waves }\left(f \leq f_{\mathrm{ce}}\right) \text {, electron } \\
\text { cyclotron waves }\end{array}$ \\
\hline 37- 05:06 UT & yes & broadband electrostatic waves $\left(f \leq f_{\mathrm{ce}}\right)$ \\
\hline 01.08.03. 05:55-09:00 UT & yes & $\begin{array}{l}\text { broadband EME }\left(f \leq f_{\text {ce }}\right) \text {, whistler waves, electron } \\
\text { cyclotron waves }\end{array}$ \\
\hline IZ-19:27 UT & yes & broadband electrostatic waves $\left(f \leq f_{\mathrm{ce}}\right)$ \\
\hline $08.08 .03 .0 .20-11: 30 \mathrm{UT}$ & yes & broadband EME $\left(f \leq f_{\text {ce }}\right)$, whistler waves \\
\hline 12.08.03. 21010 UT & no & broadband electrostatic waves $\left(f \leq f_{\mathrm{ce}}\right)$ \\
\hline 27.08.03.07:22-08:40 UT & yes & $\begin{array}{l}\text { broadband electrostatic waves }\left(f \leq f_{\text {ce }}\right) \text {, electron } \\
\text { cyclotron waves }\end{array}$ \\
\hline$-16: 30 \mathrm{UT}$ & yes & $\begin{array}{l}\text { broadband EME }\left(f \leq f_{\text {ce }}\right) \text {, whistler waves, electron } \\
\text { cyclotron waves }\end{array}$ \\
\hline 15.09.03.04:27-07:50 UT & yes & broadband electrostatic waves $\left(f \leq f_{\mathrm{ce}}\right)$ \\
\hline
\end{tabular}




\begin{tabular}{|c|c|c|}
\hline 24.09.03. 16:05 - 17:00 UT & yes & broadband electrostatic waves $\left(f \leq f_{\mathrm{ce}}\right)$ \\
\hline 29.09.03. 10:22 - 11:25 UT & yes & broadband EME $\left(f \leq f_{\mathrm{ce}}\right)$, whistler waves \\
\hline 01.10.03. 15:29-15:32 UT & yes & broadband electrostatic waves $\left(f \leq f_{\mathrm{ce}}\right)$ \\
\hline $08.10 .03 .21: 38-22: 57 \mathrm{UT}$ & yes & broadband EME $\left(f \leq f_{\mathrm{ce}}\right)$, whistler waves \\
\hline 11.10.03. 07:45-09:22 UT & yes & $\begin{array}{l}\text { broadband electrostatic waves }\left(f \leq f_{\text {ce }}\right) \text {, electron } \\
\text { cyclotron waves }\end{array}$ \\
\hline 13.10.03. 11:40-13:10 UT & yes & broadband EME $\left(f \leq f_{\mathrm{ce}}\right)$, whistler waves \\
\hline 20.10.03. 15:35 - 18:10 UT & yes & $\begin{array}{l}\text { broadband electrostatic waves }\left(f \leq f_{\text {ce }}\right) \text {, electron } \\
\text { cyclotron waves }\end{array}$ \\
\hline 03.08.04.04:10 - 04:36 UT & yes & no evident wave activity \\
\hline 14.08.04. 2:00-23:00 UT & yes & broadband EME $\left(f \leq f_{\text {ce }}\right)$, whistler waves \\
\hline $\begin{array}{l}03.10 .04 .10 .45-17: 55 \mathrm{UT} \\
-\end{array}$ & yes & $\begin{array}{l}\text { broadband electrostatic waves }\left(f \leq f_{\text {ce }}\right) \text {, electron } \\
\text { cyclotron waves }\end{array}$ \\
\hline 03.10.04. 8.57-20:08 UT & yes & $\begin{array}{l}\text { broadband EME }\left(f \leq f_{\text {ce }}\right) \text {, whistler waves, electron } \\
\text { cyclotron waves }\end{array}$ \\
\hline$-06: 05 \mathrm{UT}$ & yes & broadband EME $\left(f \leq f_{\text {ce }}\right)$, whistler waves \\
\hline 08.10.04. 16:09-16:32 UT & yes & broadband EME $\left(f \leq f_{\mathrm{ce}}\right)$, whistler waves \\
\hline 11.10.04. 11 $2130-01: 32 \mathrm{UT}$ & yes & broadband electrostatic waves $\left(f \leq f_{\text {ce }}\right)$ \\
\hline 11.10.04. 01:39-02:20 UT & yes & $\begin{array}{l}\text { broadband electrostatic waves }\left(f \leq f_{\mathrm{ce}}\right) \text {, electron } \\
\text { cyclotron waves }\end{array}$ \\
\hline 13.10 .04 . & yes & $\begin{array}{l}\text { broadband EME }\left(f \leq f_{\text {ce }}\right) \text {, whistler waves, electron } \\
\text { cyclotron waves }\end{array}$ \\
\hline$t-14: 36$ UT & yes & $\begin{array}{l}\text { broadband electrostatic waves }\left(f \leq f_{\text {ce }}\right) \text {, electron } \\
\text { cyclotron waves }\end{array}$ \\
\hline$-17: 34$ UT & yes & $\begin{array}{l}\text { broadband EME }\left(f \leq f_{\text {ce }}\right) \text {, whistler waves, electron } \\
\text { cyclotron waves }\end{array}$ \\
\hline 18:10 UT & yes & $\begin{array}{l}\text { broadband EME }\left(f \leq f_{\text {ce }}\right) \text {, whistler waves, electron } \\
\text { cyclotron waves }\end{array}$ \\
\hline $18: 33 \mathrm{UT}$ & yes & $\begin{array}{l}\text { broadband electrostatic waves }\left(f \leq f_{\text {ce }}\right) \text {, electron } \\
\text { cyclotron waves }\end{array}$ \\
\hline $44-19: 05$ UT & yes & electron cyclotron waves \\
\hline$-19: 31 \mathrm{UT}$ & yes & $\begin{array}{l}\text { broadband EME }\left(f \leq f_{\text {ce }}\right) \text {, whistler waves, electron } \\
\text { cyclotron waves }\end{array}$ \\
\hline$-20: 35 \mathrm{UT}$ & yes & electron cyclotron waves \\
\hline 07.08 .05$. & yes & $\begin{array}{l}\text { broadband EME }\left(f \leq f_{\mathrm{ce}}\right) \text {, whistler waves, electron } \\
\text { cyclotron waves }\end{array}$ \\
\hline $20-19: 32$ UT & yes & $\begin{array}{l}\text { broadband electrostatic waves }\left(f \leq f_{\text {ce }}\right) \text {, electron } \\
\text { cyclotron waves }\end{array}$ \\
\hline 17.08.05.00:38-02:16 UT & yes & $\begin{array}{l}\text { broadband electrostatic waves }\left(f \leq f_{\text {ce }}\right) \text {, electron } \\
\text { cyclotron waves }\end{array}$ \\
\hline 21.08.05 104-20:12 UT & yes & no evident wave activity \\
\hline $21.09 .05 .13-14: 19 \mathrm{UT}$ & yes & $\begin{array}{l}\text { broadband electrostatic waves and broadband } \\
\text { EME }\left(f \leq f_{\text {ce }}\right) \text {, whistler waves }\end{array}$ \\
\hline
\end{tabular}




\begin{tabular}{|c|c|c|}
\hline 21.09.05. 14:21 - 15:35 UT & yes & $\begin{array}{l}\text { broadband EME }\left(f \leq f_{\text {ce }}\right) \text {, whistler waves, electron } \\
\text { cyclotron waves }\end{array}$ \\
\hline 28.09.05. 17:31 - 20:44 UT & yes & electron cyclotron waves \\
\hline 01.10.05. 04:44 - 04:50 UT & yes & $\begin{array}{l}\text { broadband electrostatic waves }\left(f \leq f_{\text {ce }}\right) \text {, electron } \\
\text { cyclotron waves }\end{array}$ \\
\hline 01.10.05. 04:56 - 05:35 UT & yes & $\begin{array}{l}\text { broadband EME }\left(f \leq f_{\mathrm{ce}}\right) \text {, whistler waves, electron } \\
\text { cyclotron waves }\end{array}$ \\
\hline 15.10.05. 07:06 - 07:53 UT & yes & broadband electrostatic waves $\left(f \leq f_{\mathrm{ce}}\right)$ \\
\hline 17.10.05.16:07-18:30 UT & yes & electron cyclotron waves \\
\hline
\end{tabular}

Acknowleugmemts

-

We ackndwledge Cluster Science Archive (http://www.cosmos.esa.int/web/csa), PI and teams of CIS, PEA CE, GM and STAFF instruments for providing the data. The Cluster data used in this study $y$ downloaded from the Cluster Science Archive version 1.2.1 at http://ww mos.esa.int/web/csa. To obtain the data, one should start the CSA GRAPHICAL USER INTEREACE; and then to download the data, just select the particular instrument and time interval.

The work E. Grigorenko, E. A. Kronberg and P. W. Daly was supported by the Volskwagen Foundation (yant Az 90 312). P. W. Daly and E. A. Kronberg acknowledge the Deutsches Zentrum für Luft and Raumfahrt (DLR) for supporting the RAPID instrument at MPS under grant 50 OC 1401. N.Yu. Ganushkina and E.E. Grigorenko are thankful for the support received from the Academy ff Pyland as Space Cooperation in the Science and Technology Commission between Finland a leading to the results has received funding from the European Union Seventh Framework Programme (1) $7 / 2007-2013)$ under grant agreements 606716 SPACESTORM and from the European Unimn's Horizon 2020 research and innovation programme under grant agreement 637302 PROARESS. E.A. Kronberg and N.Yu. Ganushkina thanks the International Space Science 
Institute in Bern, Switzerland, for their support of the international teams on "Analysis of Cluster Inner Magnetosphere Campaign data, in application the dynamics of waves and wave-particle interaction within the outer radiation belt" and "Ring current modeling: Uncommon Assumptions and Common Misconceptions". The work of N.Yu. Ganushkina was also partly supported by NASA ayard NNX14AF34G. Work at IRAP was supported by CNRS and CNES. L.M. Zelenyi acknowled ges the Russian Academy of Science (grant P-7) and Russian Foundation for Basic Research (nraiect Nr.16-52-16009) for the support of his work.

Reference

Angelopol $\int_{\mathrm{s}}$., W. Baumjohann, C.F. Kennel, F.V. Coroniti, M.G. Kivelson, R. Pellat, R.J. Walker, H. Lii rr, G. Paschmann (1992), Bursty bulk flows in the inner central plasma sheet, J. Geophys. Res., 97, 4027-4039.

Artemyev W. Baumjohann, A. A. Petrukovich, R. Nakamura, I. Dandouras, and A. Fazakerley (2011), Proton/electron temperature ratio in the magnetotail, Ann. Geophys., 29, 2253-2257, doi:10.5194herigeo-29-2253-2011.

Ashour-Abdalla, M., Berchem, J. P., Buechner, J., and Zelenyi, L. M. (1993), Shaping of the magnetotair Irom the mantle - Global and local structuring, J. Geophys. Res., 98, 5651-5676, doi:10.1020/201662.

Ashour-A dalla, M., Frank, L. A., Paterson, W. R., Peroomian, V., and Zelenyi, L. M. (1996), Proton velocity distributions in the magnetotail: Theory and observations, J. Geophys. Res., 101, 2587-2598_do : 10.1029/95JA02539.

Balogh, et al. (2001), The Cluster magnetic field investigation: Overview of in-flight performance and initial results, Ann. Geophys., 19, 1207-1217. 
Baumjohann, W., Paschmann, G., and Cattell, C. A. (1989), Average plasma properties in the central plasma sheet, J. Geophys. Res., 94, 6597-6606, doi:10.1029/JA094iA06p06597.

J. Birn, M. Hesse, R. Nakamura, S. Zaharia (2013) Particle acceelration in dipolarization events, J. Geophys. Res., 118, 1960-1971, doi:10.1002/jgra.50132.

J. Birn, A. Runov and M. Hesse (2014), Energetic electrons in dipolarization events: Spatial properties and nisotropy, J. Geophys. Res., 119, 3604-3616, doi: 10.1002/2013JA019738.

Biskamp (2000), Magnetic Reconnection in Plasmas, Cambridge Univ. Press, New York.

Cornilleau-wenrlin N. et al. (2003), First results obtained by the Cluster STAFF experiment, Ann. Geophys., $2437-456$.

()

Daly, P. W. and E. A. Kronberg (2015), User guide to the RAPID measurements in the Cluster Active Arcmive (CAA), Tech. Rep. CAA-EST-UG-RAP, European Space Agency, Paris.

Deng X., M. Ashour-Abdalla, M. Zhou, R. Walker, M. El-Alaoui, V. Angelopoulos, R.E. Ergun, D. Schriver 201), Wave and particle characteristics of earthward injections associated with dipolarizations fonts, J. Geophys. Res., 115, A09225, doi:10.1029/2009JA015107.

Drake J.F., M. Swisdak, H. Che, M.A. Shay (2006), Electron acceleration from contracting magnetic lands during reconnection, Nature 443, 553-556, doi:10.1038/nature05116.

Farrell WM., M.D. Desch, K.W. Ogilvie, M.L. Kaiser, and K. Goetz (2003), The role of upper hybrid waves in magnetic reconnection, Geophys. Res. Lett., 30(24),2259, doi:10.1029/2003GL017549.

Fu, H. S., suprath electrons behind dipolarization fronts, Geophys. Res. Lett., 38, L16104, doi:10.1029/2011GL048528. 
Fu, H. S., Y. V. Khotyaintsev, A. Vaivads, M. André, V. A. Sergeev, S. Y. Huang, E. A. Kronberg, and P. W. Daly (2012a), Pitch angle distribution of suprathermal electrons behind dipolarization fronts: A statistical overview, J. Geophys. Res., 117, A12221, doi:10.1029/2012JA018141.

Fu, H. S., Y. V. Khotyaintsev, A. Vaivads, M. André, and S. Y. Huang (2012b), Occurrence rate of earthward-propagating dipolarization fronts, Geophys. Res. Lett., 39, L10101, doi:10.1029/20 2GL051784.

Fu, H. S.y. Khotyaintsev, A. Vaivads, A. Retino, and M. Andre (2013a), Energetic electron acceleration by unsteady magnetic reconnection, Nature Physics, 9, 426-430, doi:10.103 hys 2664 .

Fu, H. S., (2013b), Dipolarization fronts as a consequence of transient reconnection: In situ evidence, Geop yys. Res. Lett., 40, 6023-6027, doi:10.1002/2013GL058620.

Fu, H. S., t al. (2014), Whistler-mode waves inside flux pileup region: Structured or unstructured?, J. Geoph Res. Space Physics, 119, 9089-9100, doi:10.1002/2014JA020204 Gurnett_D.A. Frank, L. A., Lepping, R. P., et al.: Plasma waves in the distant magnetotail, J. Geophys. $81,6059-6071,1976$.

Hoshino M. (2005), Electron surfing acceleration in magnetic reconnection. J. Geophys. Res. 110 (A9), A10zT, doi:10.1029/2005JA011229.

Hoshino T. Tukai, T. Yamamoto, S. Kokubun (1998), Ion dynamics in magnetic reconnection: Comparis $\mathrm{n}$ between numerical simulation and Geotail observations, J. Geophys. Res., 103, A3, 4509-45su.

Hoshino, M. Hiraide, and T. Mukai (2001), Strong electron heating and non-Maxwellian behaviorir agnetic reconnection, Earth Planets Space, 53, 627- 634. 
Hwang K.-J., M.L. Goldstein, E. Lee, J.S. Pickett (2011), Cluster observations of multiple dipolarization fronts, J. Geophys. Res., 116, A00132, doi:10.1029/2010JA015742.

Johnstone, A.D., et al. (1997), PEACE: A plasma electron and current experiment, Space Sci. Rev., 79, 351-398.

Imada S., R. Nakamura, P. W. Daly, M. Hoshino, W. Baumjohann, S. Mühlbachler, A. Balogh, and H. Réme Eng getic electron acceleration in the downstream reconnection outflow region, J. Geophys. Pec 112, A03202, doi:10.1029/2006JA011847, 2007

Kaufmann, K. L., Paterson, W. R., and Frank, L. A. (2005), Relationships between the ion flow speed, masedc flux transport rate, and other plasma sheet parameters, J. Geophys. Res., 110, A09216, d. .1029/2005JA011068.

Khotyaintsev Ju.V., C. M. Cully, A. Vaivads, and M. André (2011), Plasma Jet Braking: Energy Dissipatio and Nonadiabatic Electrons, PRL, 106, DOI: 10.1103/PhysRevLett.106.165001.

Kronberg E.E. Grigorenko, S. E. Haaland, P. W. Daly, D. C. Delcourt, H. Luo, L. M. Kistler and I. Dandouras (2015), Distribution of energetic oxygen and hydrogen in the near-Earth plasma sheet, J. Gepinys. Res., Vol. 120, 10.1029/2014JA020882.

Lavraud B., J. E. Borovsky, V. Génot, S. J. Schwartz, J. Birn, A. N. Fazakerley, M. W. Dunlop, M. G. G. T. Fayror, H. Hasegawa, A. P. Rouillard, J. Berchem, Y. Bogdanova, D. Constantinescu, I. Dandouras Eastwood, C. P. Escoubet, H. Frey, C. Jacquey, E. Panov, Z. Y. Pu, C. Shen, J. Shi, D. G. Sibeck, M. Volwerk, and J. A. Wild (2009), Tracing solar wind plasma entry into the magnetosynere using ion-to-electron temperature ratio, Gephys. Res. Lett., 36, L18109, doi:10.1029/20)9GL039442

Le Conter et al. (2009), Quasi-parallel whistler mode waves observed by THEMIS during nearEarth dipoalrizations, Ann. Geophys., 27, 2259 - 2275. 
Lui A.T.Y. (2004), Potential plasma instabilities for substorm expansion onsets, Space Science Reviews, 113, 127-206.

Lui A.T.Y., P.H. Yoon, C. Mok and C.-M. Ryu (2008), Inverse cascade feature in current disruption, J. Geophys. Res., 113, A00C06, doi:10.1029/2008JA013521.

Lyons, L. B. (1984), Electron energization in the geomagnetic tail current sheet, J. Geophys. Res., 89, 5479-5487, doi:10.1029/JA089iA07p05479.

Matsumøt Kojima, Y. Kasaba, T. Miyake, Y. Omura, M. Okada, I. Nagano, M. Tsutsui (1994), Electrostatic solitary waves (ESW) in the magnetotail: BEN wave forms observed by GEOTAIL,

Nakamura. al. (2002), Motion of the dipolarization front during a flow burst event observed by Cluster, Geoph s. Res. Lett., 29(20), 1942, doi:10.1029/2002GL015763.

R. Nakan ara, A Retinò, W. Baumjohann, M. Volwerk, N. Erkaev, B. Klecker, E.A. Lucek, I. Dandouras André, Y. Khotyaintsev (2009), Evolution of dipolarization in the near_earth current sheet indiced by Earthward rapid flux transport, Ann. Geophys., 27, 1743-1754.

Ohtani S.L.A. Shay, T. Mukai (2004), Temporal structure of the fast convective flow in the plasma sheet: Comparison between observations and two-fluid simulations, J. Geophys. Res., 109, A03210, dor.10.1029/2003JA10002.

Oka M., Dhan, S. Krucker,M. Fujimoto, I. Shinohara, Electron acceleration by multi-island coalescende(2010), Astrophys. J., 714, 915-926, doi:10.1088/0004-637X/714/1/915.

Petkaki Frive. Freeman, A.P. Walsh (2006), Cluster observations of broadband electromagnetic waves in and ound a reconnection region in the Earth's magnetotail current sheet, Geophys. Res. Lett., 33 105, doi:10.1029/2006GL027066. 
Réme, H., C. Aoustin, J. M. Bosqued, I. Dandouras et al. (2001), First multispacecraft ion measurements in and near the Earth's magnetosphere with identical Cluster ion spectrometry (CIS) experiment, Ann. Geophys., 19, 1303.

Runov A., V. Angelopoulos, M.I. Sitnov, V.A. Sergeev, J. Bonnell, J.P. McFadden, D. Larson, K. Glassmerter (2009), THEMIS observations of an earthward-propagating dipolarization front. Geohys Res. Lett. 36, L14106, doi:10.1029/2009GL038980.

Runov A. A. Angelopoulos, C. Gabrielse, J. Liu. D.L. Turner, X.-Z. Zhou (2015), Average thermodynamy and spectral properties of plasma in and around dipolarizing flux bundles, J. Geophys. Res 120, 4369-4383, doi:10.1002/2015JA021166.

Sergeev V.A., V. Angelopoulos, J.T. Gosling, C.A. Cattel, C.T. Russell (1996), Detection of localized prasma-depleted flux tubes or bubbles in the midtail plasma sheet, J. Geophys. Res., 101, 10,817-10

Sergeev, A. V. Angelopoulos, and R. Nakamura (2012), Recent advances in understanding substormevnamics, Geophys. Res. Lett., 39, L05101, doi:10.1029/2012GL050859.

V.A. S N.P. Dmitrieva, N.A. Stepanov, D.A. Sormakov, V. Angelopoulos, A.V. Runov (2015), Qn the plasma sheet dependence on solar wind and substorms and its role in magnetosphere ionosphere coupling, Earth, Planets and Space, 67:133, doi:10.1186/s40623-0150296-x.

Sitnov vir., Swisdak, A.V. Divin (2009), Dipolarization fronts as a signature of of transient reconnection in the magnetotail, J. Geophys. Res., 114, A04202, doi:10.1029/2008JA013980.

Sharma S., R. Nakamura, A. Runov, E. E. Grigorenko, H. Hasegawa, M. Hoshino, P. Louarn, C. J. Owen, A. trukovich, J.-A. Sauvaud, V. S. Semenov, V. A. Sergeev, J. A. Slavin, B. U. Ö. 
Sonnerup, L. M. Zelenyi, G. Fruit, S. Haaland, H. Malova, and K. Snekvik (2008), Transient and Localized Processes in the Magnetotail: A Review, Annales Geophysicae, 26, 955-1006.

Shiokawa K., W. Baumjohann, G. Haerendel (1997), Braking of high-speed flows in the near-Earth tail, Geophys. Res. Lett., 24, 1179-1182, doi:10.1029/97GL01062.

Viberg $\mathrm{H}_{\mathbf{3}} \mathrm{Yu}$ V. Khotyaintsev, A. Vaivads, M. André, H.S. Fu, N. Cornilleau-Wehrlin (2104),

Whistler 100 waves at magnetotail dipolarization fronts, J. Geophys. Res., 119, 2605-2611, doi:10.1@020014JA019892.

Wang C.-P., Mr. Gkioulidou, L.R. Lyons, V. Angelopoulos (2012), Spatial distributions of the ion to electron terpature ratio in the magnetosheath and plasma sheet, J. Geophys. Res., 117, A08215, doi: 10.1062 2JA017658.

Wilken, B., et 1. (2001), First results from the RAPID imaging energetic particle spectrometer on board Clu ter, Ann. Geophys., 19, 1355-1366.

Zelenyi L tail of the magnetosphere, Cosmic Res. (English edition), 28, 3, 369-381.

Zhang Angelopoulos (2014), On the relationship of electrostatic cyclotron harmonic emissions with electron injections and dipolarization fronts, J. Geophys. Res., 119, 2536-2549, doi:10.10Gz/ZOIJJA019540.

Zhou M., Ashour-Abdalla, X. Deng, D. Schriver, M. El-Alaoui, Y. Pang (2009), THEMIS observatio of multiple dipolarization fronts and associated wave characteristics in the near-Earth magnetotan, Ueophys. Res. Lett., 36, L20107,doi:10.1029/2009GL040663.

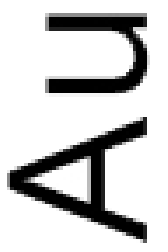


Figure Captions

Figure 1. The scatterplots of the PS samples using in our studies in the $(Y Z)$ and $(X Y)$ planes.

Figure 2. From,top to bottom: (a): the statistical distribution of the average values of $\left\langle T_{p} / T_{e}\right\rangle$ in the $(X Y)$ plan $1 \mathrm{~T})<T_{p} / T_{e}>$ were averaged over $2 \mathrm{R}_{\mathrm{E}} \times 2 \mathrm{R}_{\mathrm{E}}$ bin. The colored scale in the right part of the Figuredienlays the values of $\left\langle T_{p} / T_{e}\right\rangle$. (b): the $\left\langle T_{p} / T_{\mathrm{e}}\right\rangle(R)$ profile integrated over all $Y$ locations for a given $K$-oin. (c): the $\left\langle T_{p} / T_{\mathrm{e}}\right\rangle(Y)$ profile integrated over all $R$ locations for a given $Y$-bin. (d): a histogram occurrence frequency distribution of the $T_{p} / T_{e}$ observed in the PS samples from our data base.

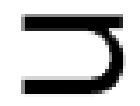

Figure 3. example of the PS crossing by Cl-1 On 8.10.2001, in which the decreases of $T_{p} / T_{e}$ below 3.5 observed. From top to bottom: the Energy-Time spectrograms of omni-directional electrons (PEACE data) (a) and protons (CODIF data) (b); the time profiles of the $T_{\mathrm{p}}$ (black line) and $T_{\mathrm{e}}$ (redvite) (c); $T_{\mathrm{p}} / T_{\mathrm{e}}(\mathrm{d}) ;$ and $\mathrm{T}_{\mathrm{PAR}} / \mathrm{T}_{\mathrm{PER}}$ fro protons (black line) and electrons (red line) (e); the three components of the magnetic field (f); the frequency spectra of the electric $|\mathrm{E}|(\mathrm{g})$ and magnetic $|\mathbf{B}|$ (n) field fluctuations in frequency range $8-4096 \mathrm{~Hz}$; the time-frequency distribution of the val angle between the Poynting flux of electromagnetic fluctuations and the ambient magnetic feld (i) obtained by STAFF experiment. The time profiles of $f_{c e}, f_{p p}$ and $f_{L H}$ are shown by the white,magenta and black lines respectively in panels $(\mathrm{g}, \mathrm{h})$ and by the purple, magenta and black lines respective $y$ in panel (i). 
Figure 4. The zoom of the interval "II", in which the minimum of $T_{\mathrm{p}} / T_{\mathrm{e}}$ was observed (see Figure 3). From top to bottom: time profiles of $X$-component of proton bulk velocity (a) of proton (in black) and of electron (in red) $T_{\|}$and $T_{\perp}$ (b); the variations of electron temperature $\Delta T_{e}$ observed by PEACE instrument (in red) and the variations of electron temperature expected from the betatron heating $\Delta T_{\text {man }}$ (in black) (c); the time profile of the magnetic fluctuations power $\delta B^{2}$ integrated in $\left[f_{\mathrm{pp}} f_{\mathrm{ce}}\right]$ requency range $(\mathrm{d})$; the time profile of $T_{\mathrm{p}} / T_{\mathrm{e}}(\mathrm{e})$; three components of the magnetic field (f) and STAFF observations in the same format as in Figure 3 (g-i).

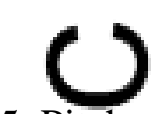

Figure 5. Pjtch angle distributions (PAD) of electrons and the spectra of electric $|E|$ and magnetic $|B|$ field fluctuations observed in three time periods ("1-3") marked by the blue horizontal lines in Figure 4.

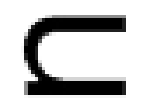

Figure 6. hor sults of epoch superposition analysis applied to the intervals listed in Tab.1 (see the Appendirom top to bottom: the epoch profiles of $A E(t) ; \beta(t) ; V_{X}(t) ; T_{\mathrm{p}}(t) ; T_{\mathrm{e}}(t) ;\left[T_{\mathrm{p}} / T_{\mathrm{e}}\right](t)$ and the $B_{Z}{ }^{*}$ nnd power of electric and magnetic field fluctuations integrated with the frequency range $\left[f_{p p}, f_{c e}\right]$. The red dashed line indicates the onset of dipolarization in each event from our data base. The parizontal blue line shows the "turbulent" phase of dipolarizations. The scatterplots of low and upper quartiles of the corresponding data sets used for the epoch analysis are displayed by the the greycurs

Figure 7 scatterplot of variation $\Delta T_{\mathrm{e}}$ versus $\Delta T_{\mathrm{p}}$ at the moments of minimum $T_{\mathrm{p}} / T_{\mathrm{e}}$ observation in the PS intoralls listed in Tab.1. 
Figure 8. Left column: scatterplots of $T_{\mathrm{e}}$ versus the power of electric and magnetic field fluctuations integrated with the frequency range $\left[f_{p p}, f_{c e}\right]$ observed during the PS intervals listed in Tab.1. Right column: the same for $T_{\mathrm{p}} / T_{\mathrm{e}}$.

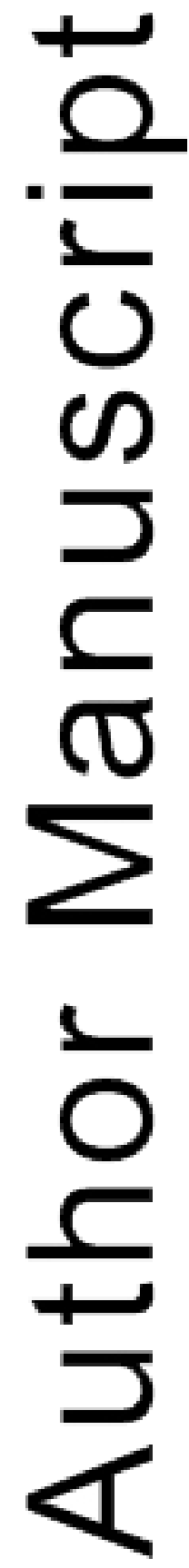

This article is protected by copyright. All rights reserved. 

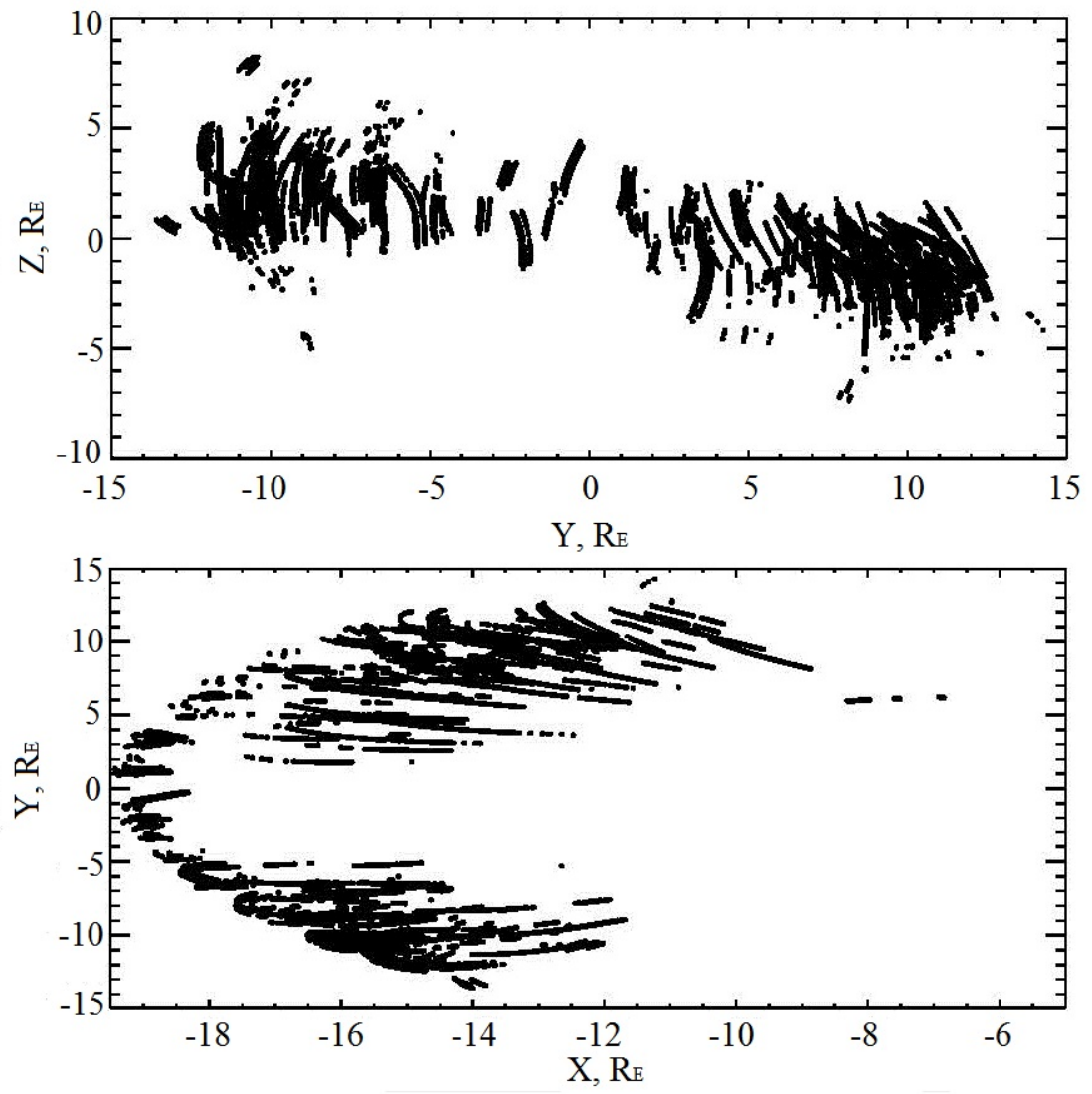

Figure 1. The scatterplots of the PS samples using in our studies in the $(Y Z)$ and $(X Y)$ planes.
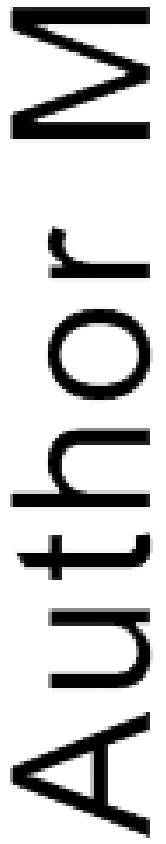

This article is protected by copyright. All rights reserved. 

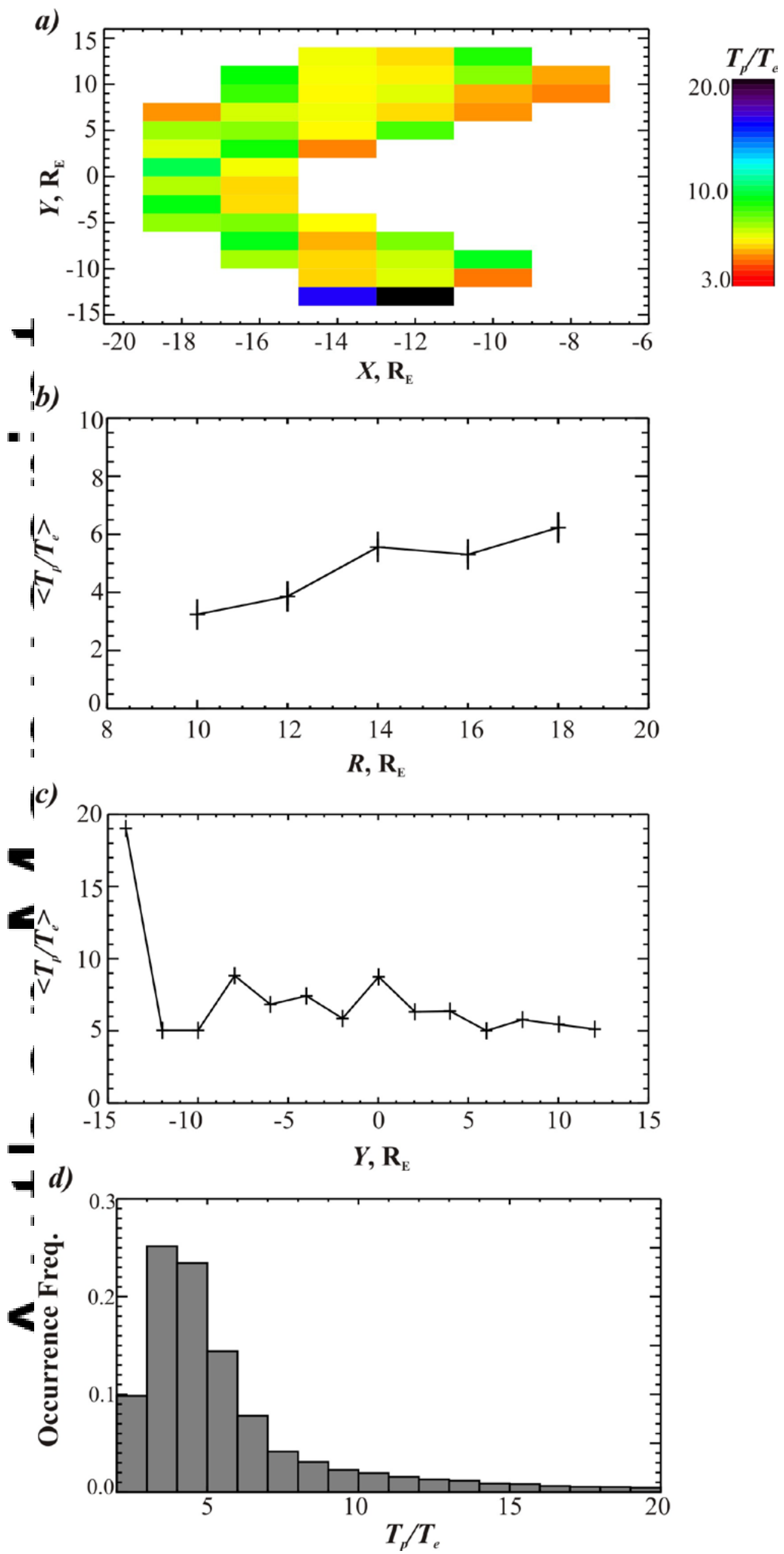
Figure 2. From top to bottom: (a): the statistical distribution of the average values of $\left\langle T_{p} / T_{e}\right\rangle$ in the $(X Y)$ plane. The $\left\langle T_{p} / T_{e}\right\rangle$ were averaged over $2 \mathrm{R}_{\mathrm{E}} \times 2 \mathrm{R}_{\mathrm{E}}$ bin. The colored scale in the right part of -

the Figure diand ays the values of $\left\langle T_{p} / T_{e}\right\rangle$. (b): the $\left\langle T_{p} / T_{\mathrm{e}}\right\rangle(R)$ profile integrated over all $Y$ locations for a given $R$-bin. (c): the $\left\langle T_{p} / T_{\mathrm{e}}\right\rangle(Y)$ profile integrated over all $R$ locations for a given $Y$-bin. (d): a histogram occurrence frequency distribution of the $T_{p} / T_{e}$ observed in the PS samples from our data base.

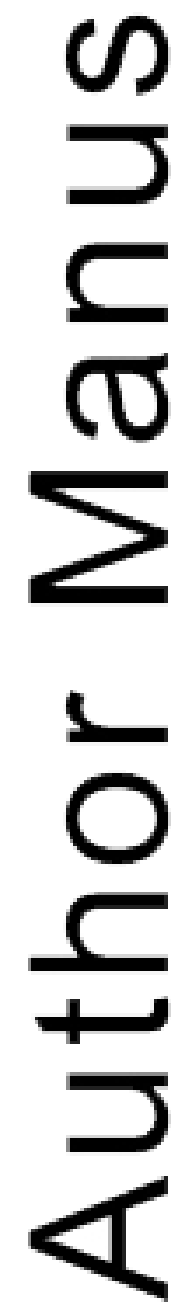

This article is protected by copyright. All rights reserved. 


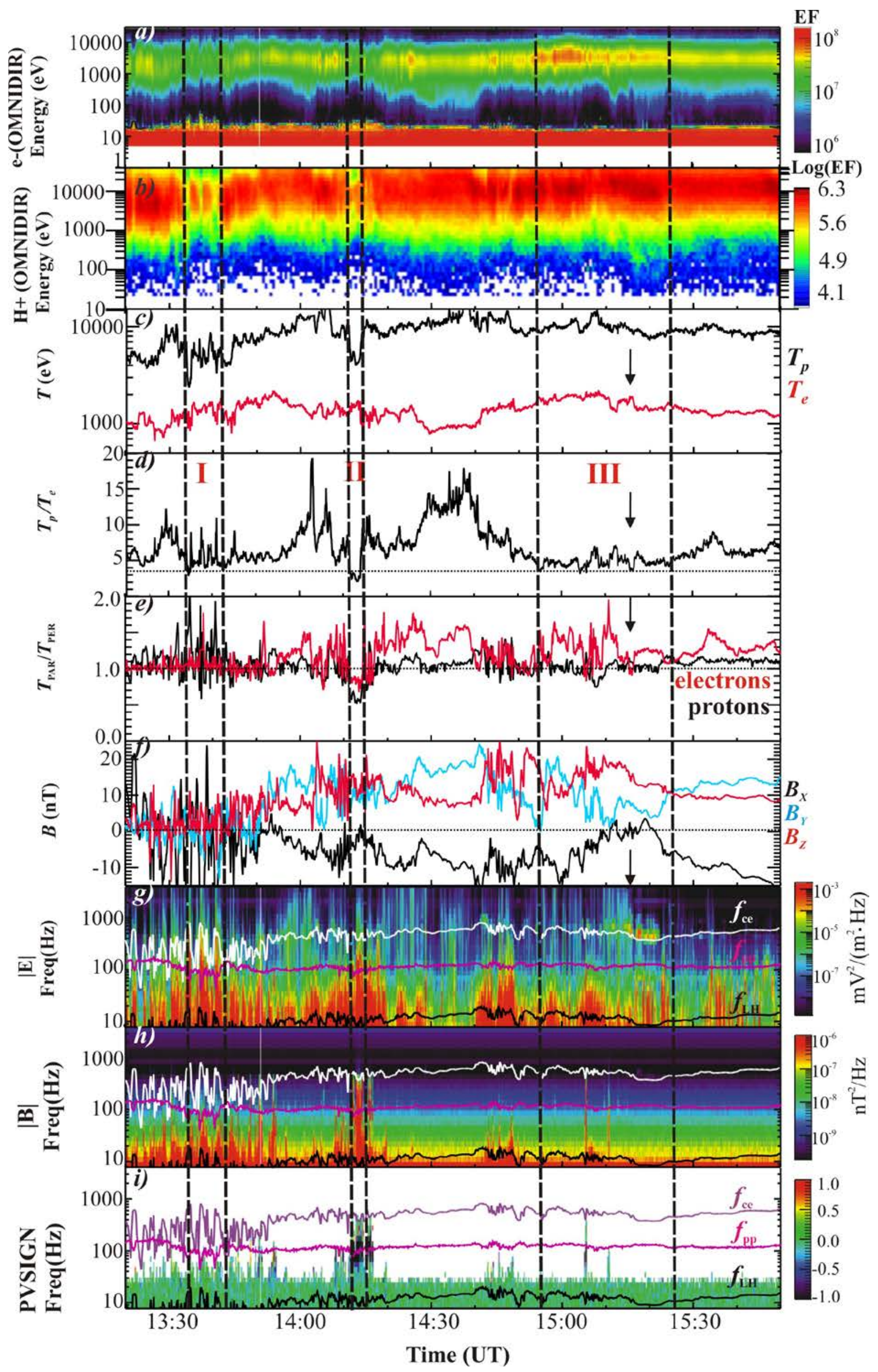


Figure 3. An example of the PS crossing by Cl-1 On 8.10.2001, in which the decreases of $T_{p} / T_{e}$ below 3.5 were observed. From top to bottom: the Energy-Time spectrograms of omni-directional y.trer

electrons (PEA CE data) (a) and protons (CODIF data) (b); the time profiles of the $T_{\mathrm{p}}$ (black line) and $T_{\mathrm{e}}$ (red line) (c); $T_{\mathrm{p}} / T_{\mathrm{e}}(\mathrm{d}) ;$ and $\mathrm{T}_{\mathrm{PAR}} / \mathrm{T}_{\mathrm{PER}}$ fro protons (black line) and electrons (red line) (e); the three magnetic 10 field fluctuations in frequency range $8-4096 \mathrm{~Hz}$; the time-frequency distribution of the valuangle between the Poynting flux of electromagnetic fluctuations and the ambient magnetic field i) obtained by STAFF experiment. The time profiles of $f_{c e}, f_{p p}$ and $f_{L H}$ are shown by the white, ragenta and black lines respectively in panels $(\mathrm{g}, \mathrm{h})$ and by the purple, magenta and black lines respectively in panel (i).

(6)
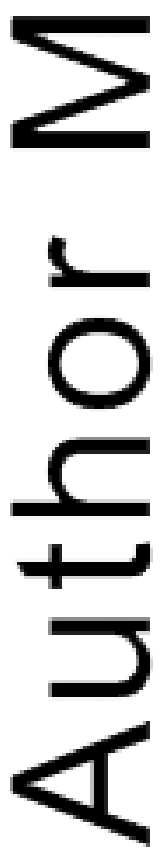


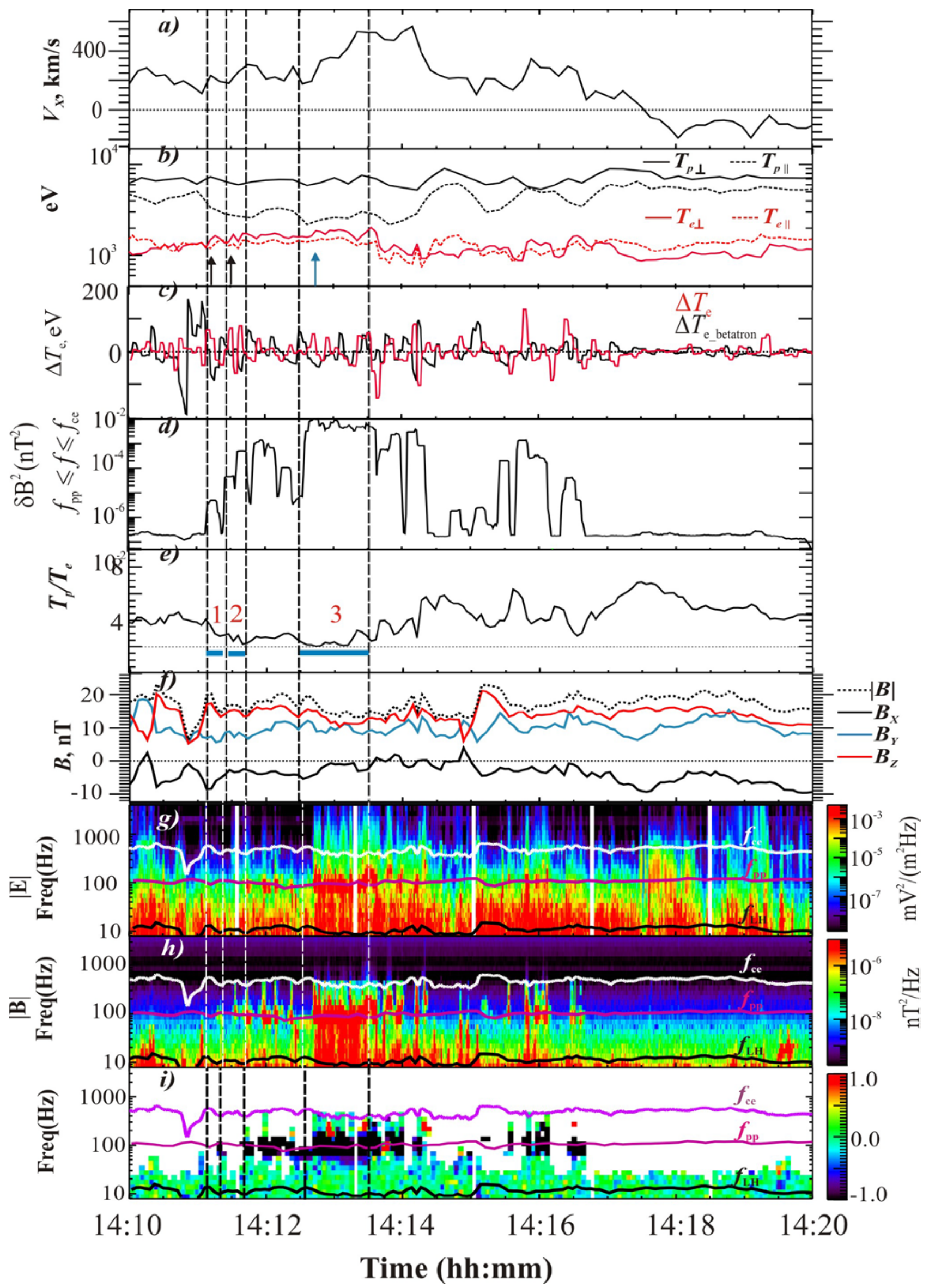


Figure 4. The zoom of the interval "II", in which the minimum of $T_{\mathrm{p}} / T_{\mathrm{e}}$ was observed (see Figure 3). From top to bottom: time profiles of $X$-component of proton bulk velocity (a) of proton (in black) and of electron (in red) $T_{\|}$and $T_{\perp}$ (b); the variations of electron temperature $\Delta T_{e}$ observed by PEACE instrument (in red) and the variations of electron temperature expected from the betatron heating $\Delta \boldsymbol{T} \boldsymbol{m}_{\text {iron }}$ (in black) (c); the time profile of the magnetic fluctuations power $\delta B^{2}$ integrated in $\left[f_{\mathrm{pp}}, f_{\mathrm{ce}}\right]$ requency range $(\mathrm{d})$; the time profile of $T_{\mathrm{p}} / T_{\mathrm{e}}(\mathrm{e})$; three components of the magnetic field (f) and STAFF observations in the same format as in Figure 3 (g-i).

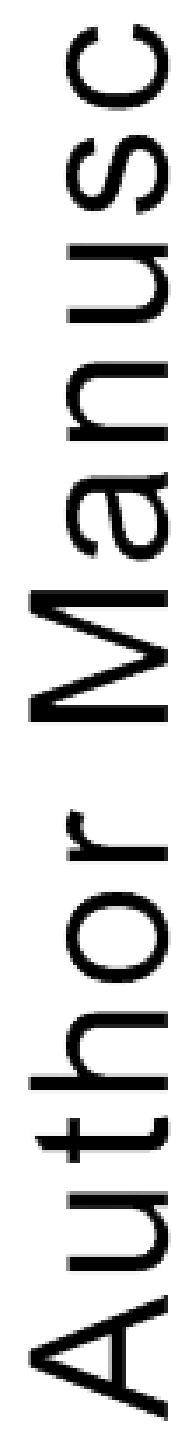

This article is protected by copyright. All rights reserved. 

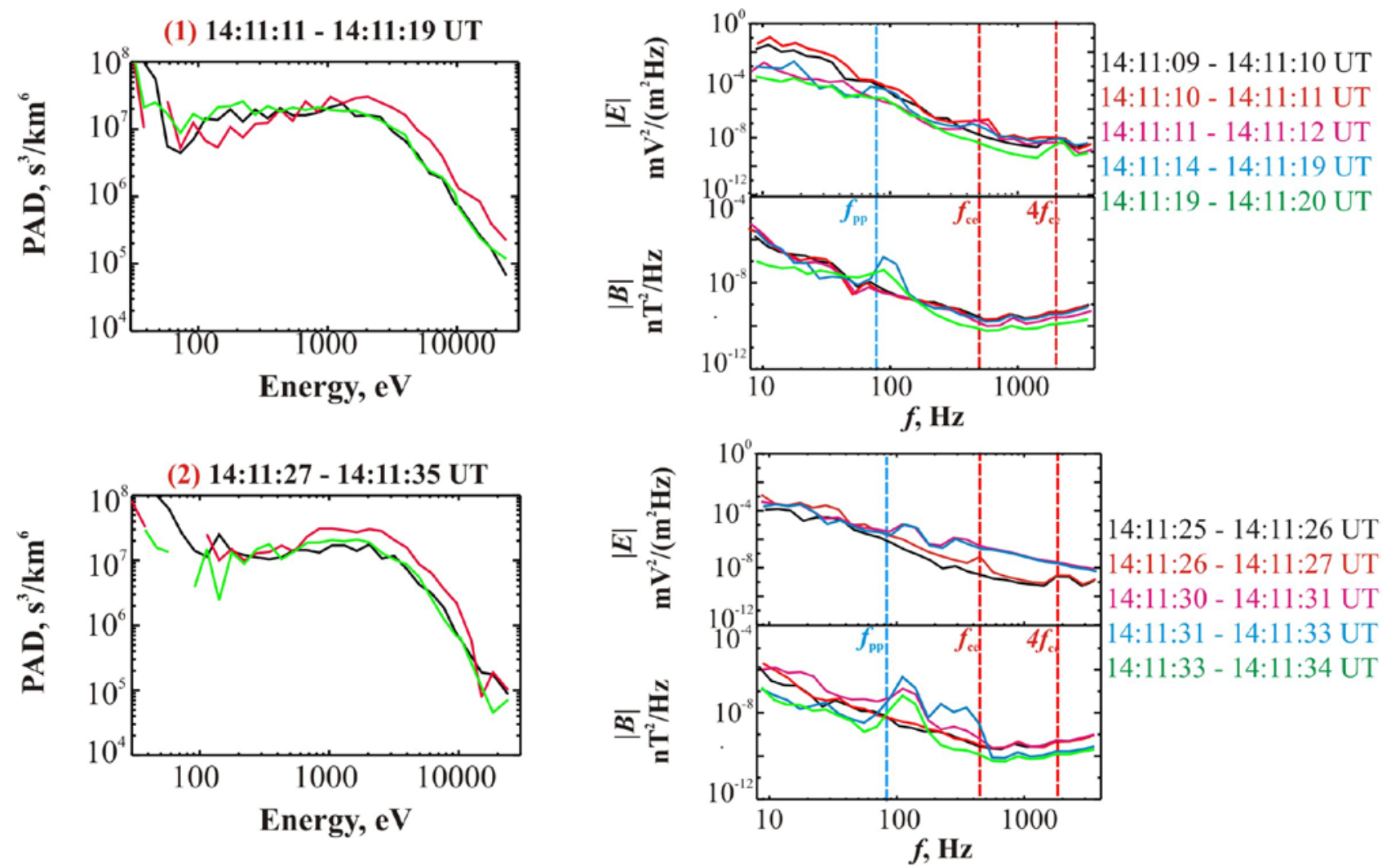

(3) 14:12:41 - 14:13:37 UT
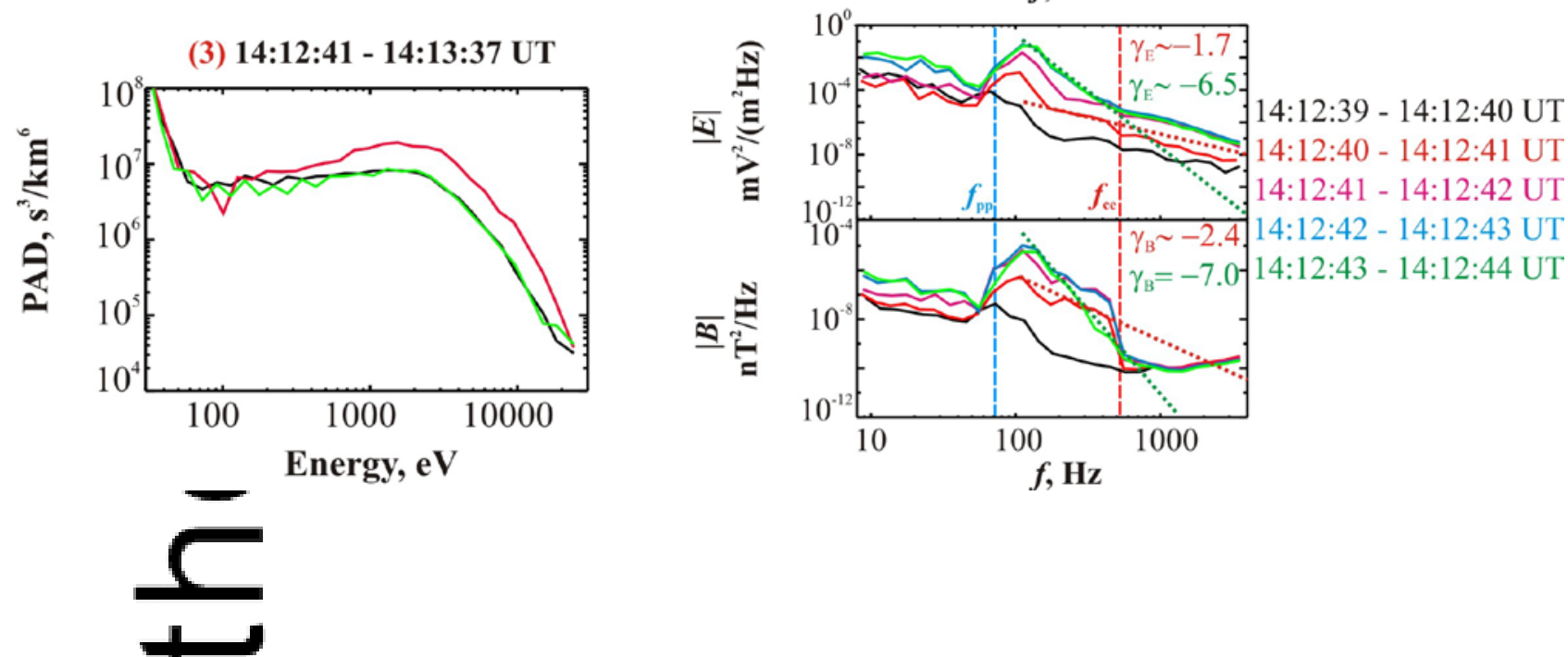

Figure 5. Pitch angle distributions (PAD) of electrons and the spectra of electric $|E|$ and magnetic $|B|$ field fluctuatrons observed in three time periods ("1-3") marked by the blue horizontal lines in Figure 


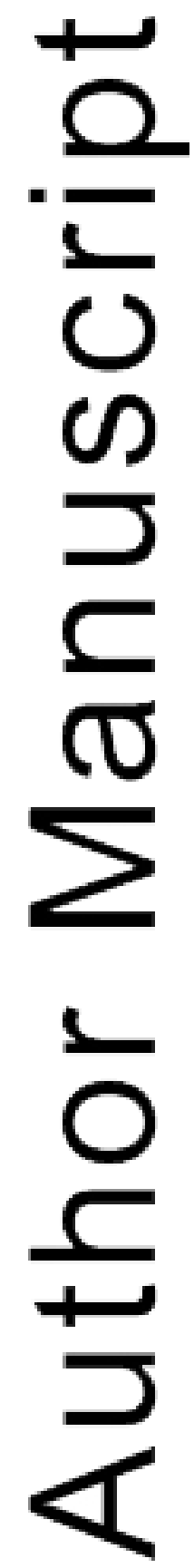

This article is protected by copyright. All rights reserved. 


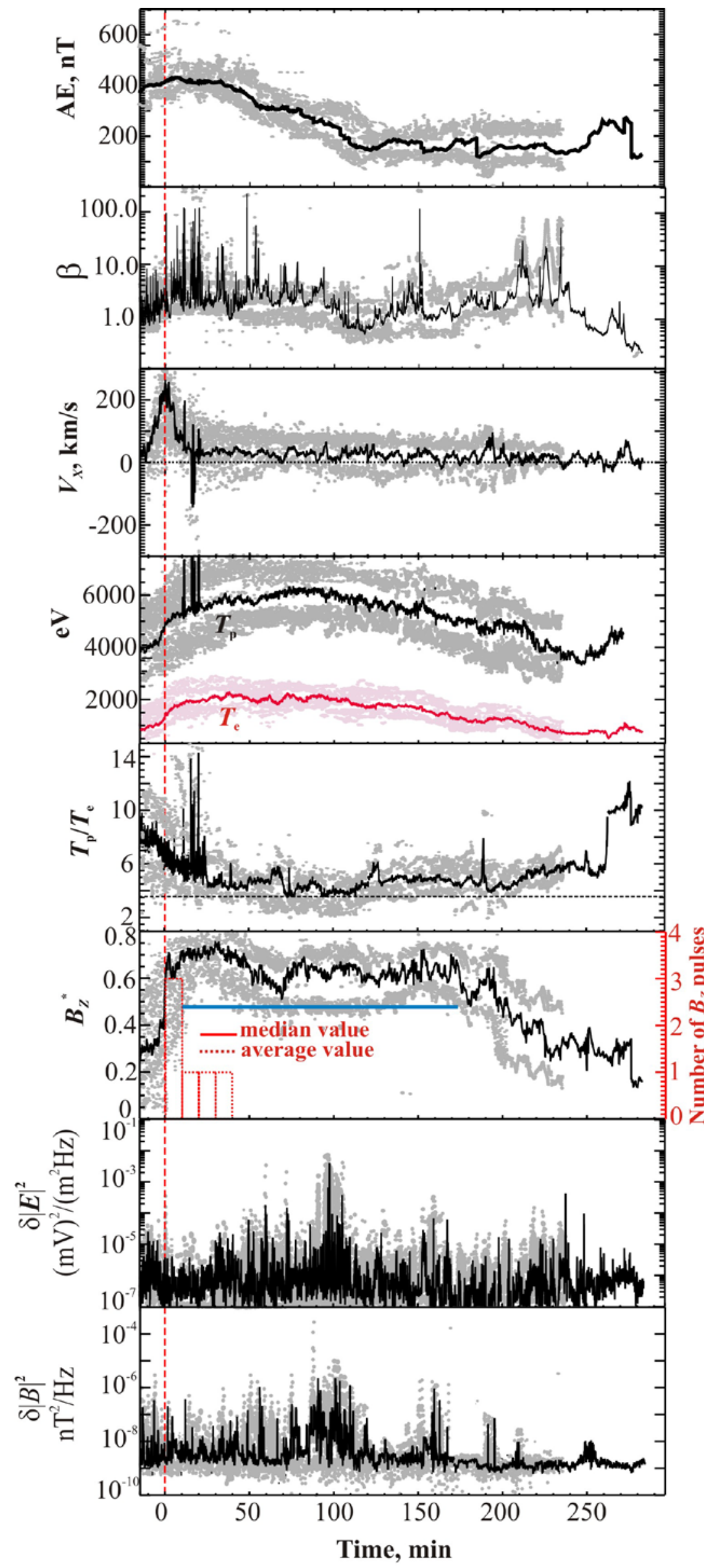

Figure 6. The results of epoch superposition analysis applied to the intervals listed in Tab.1 (see the Appendix). From top to bottom: the epoch profiles of $A E(t) ; \quad \beta(t) ; \quad V_{X}(t) ; \quad T_{\mathrm{p}}(t) ; \quad T_{\mathrm{e}}(t) ;$ $\left[T_{\mathrm{p}} / T_{\mathrm{e}}\right](t)$ and the $B_{Z}{ }^{*}(t)$ and the power of electric and magnetic field fluctuations integrated with the frequency range $\left[f_{p p}, f_{c e}\right]$. The red dashed line indicates the onset of dipolarization in each event from our data base. The horizontal blue line shows the "turbulent" phase of dipolarizations. The scatterplots of low and upper quartiles of the corresponding data sets used for the epoch analysis are displayed by the grey dots 


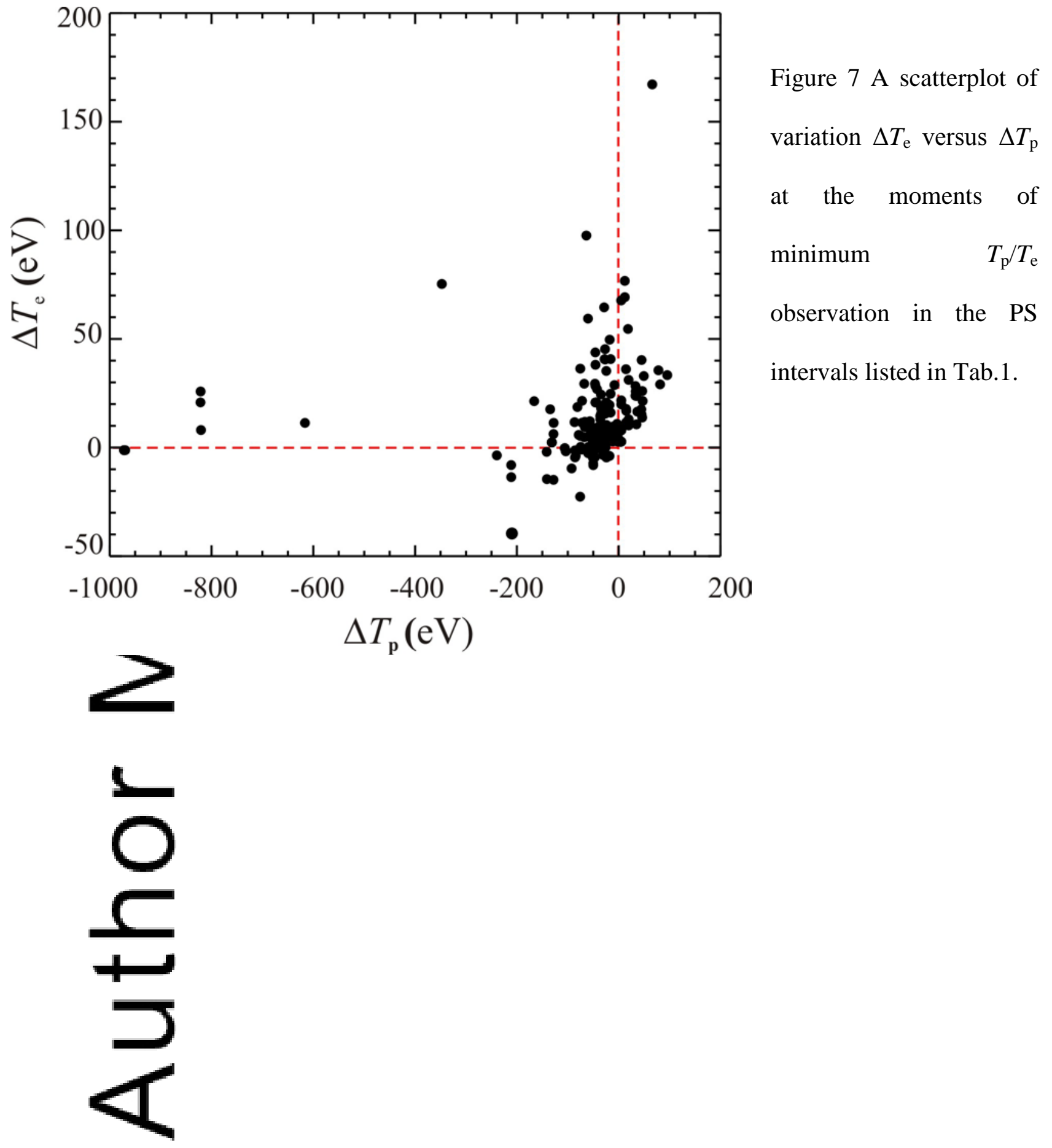

This article is protected by copyright. All rights reserved. 

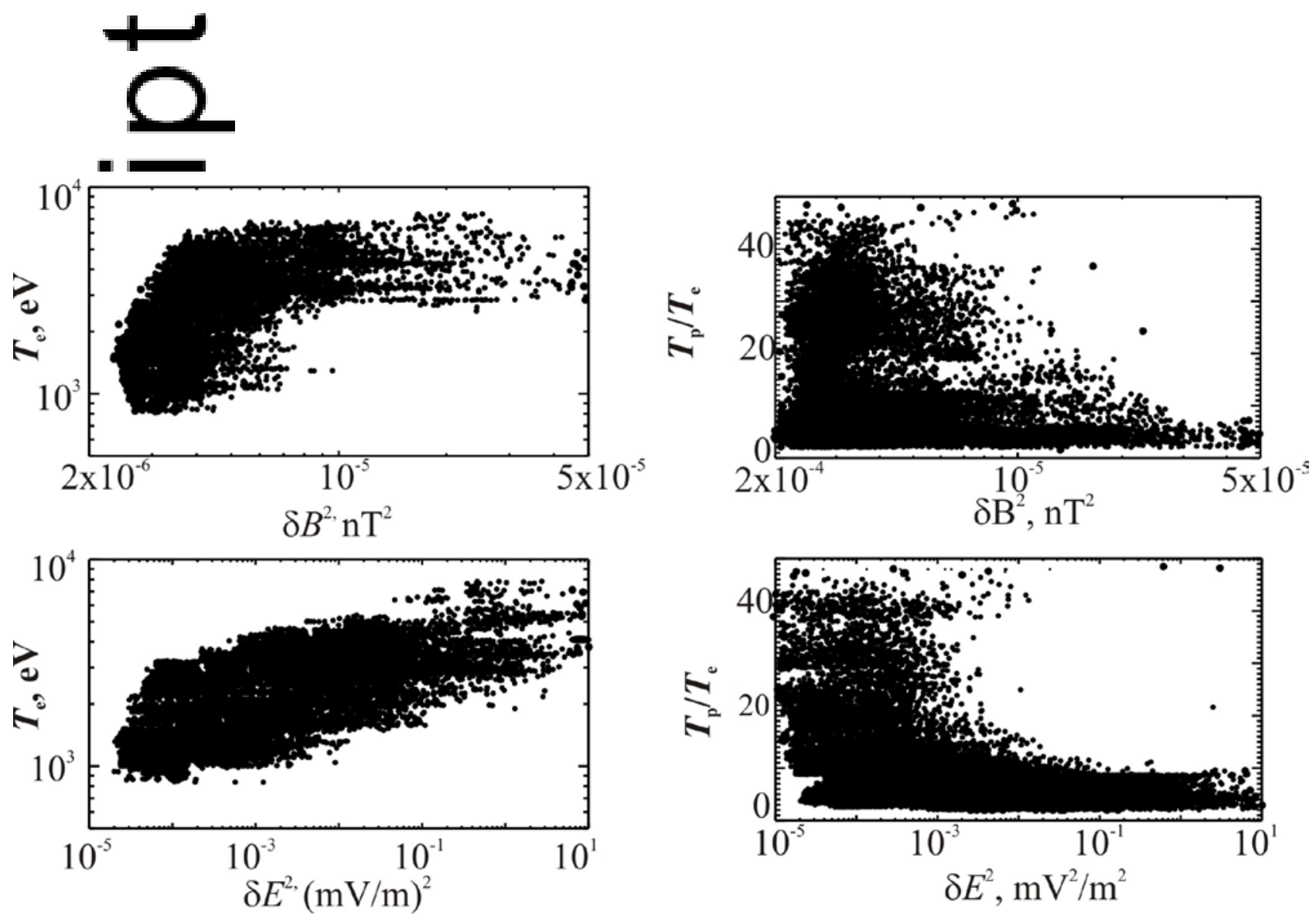

Figure 8. Left column: scatterplots of $T_{\mathrm{e}}$ versus the power of electric and magnetic field fluctuations integratea witu the frequency range $\left[f_{p p}, f_{c e}\right]$ observed during the PS intervals listed in Tab.1. Right column: the same for $T_{\mathrm{p}} / T_{\mathrm{e}}$.

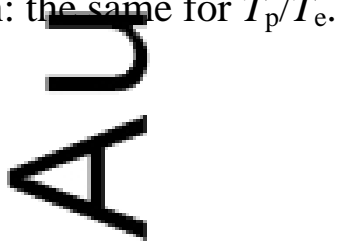




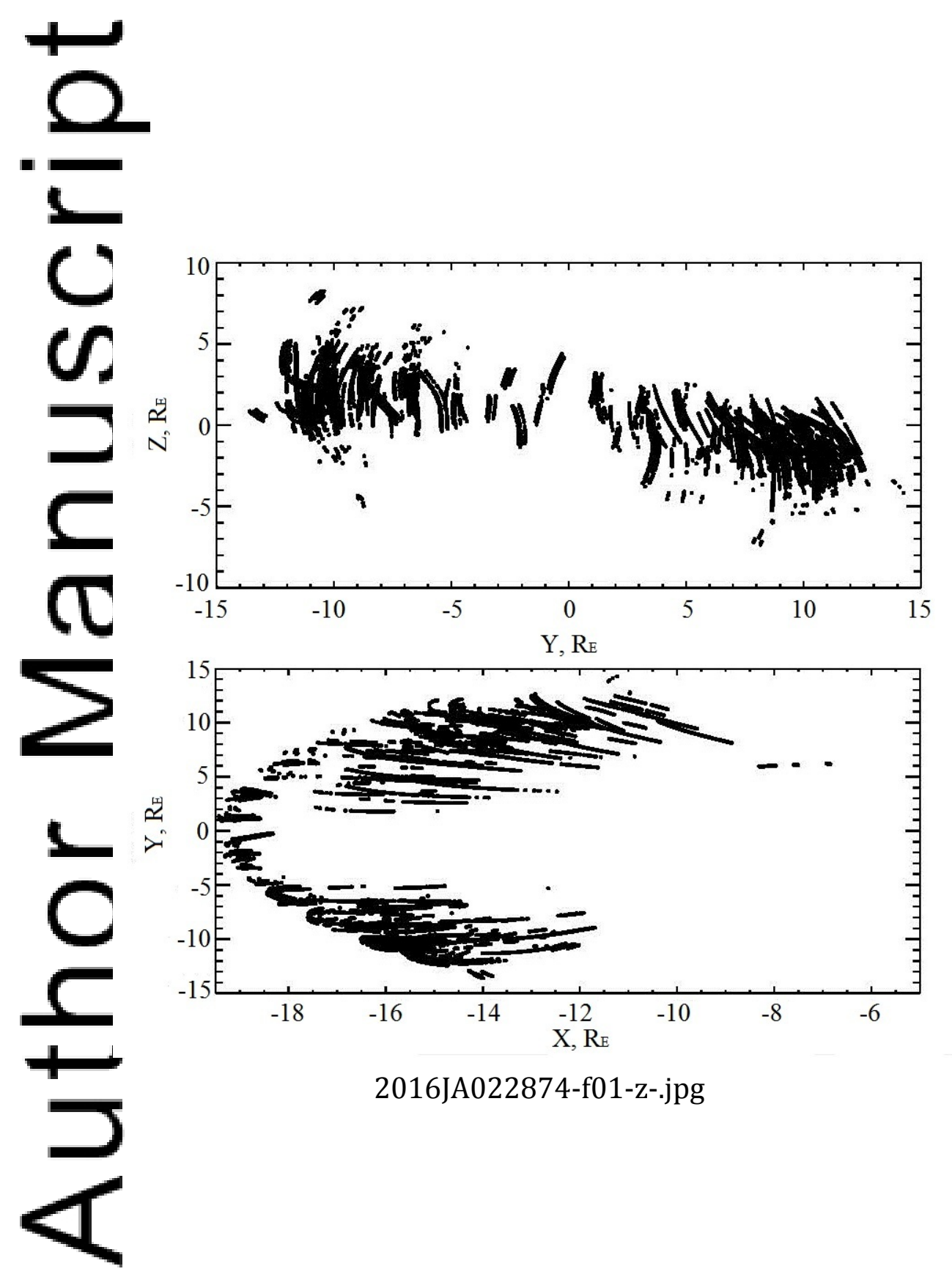

This article is protected by copyright. All rights reserved. 


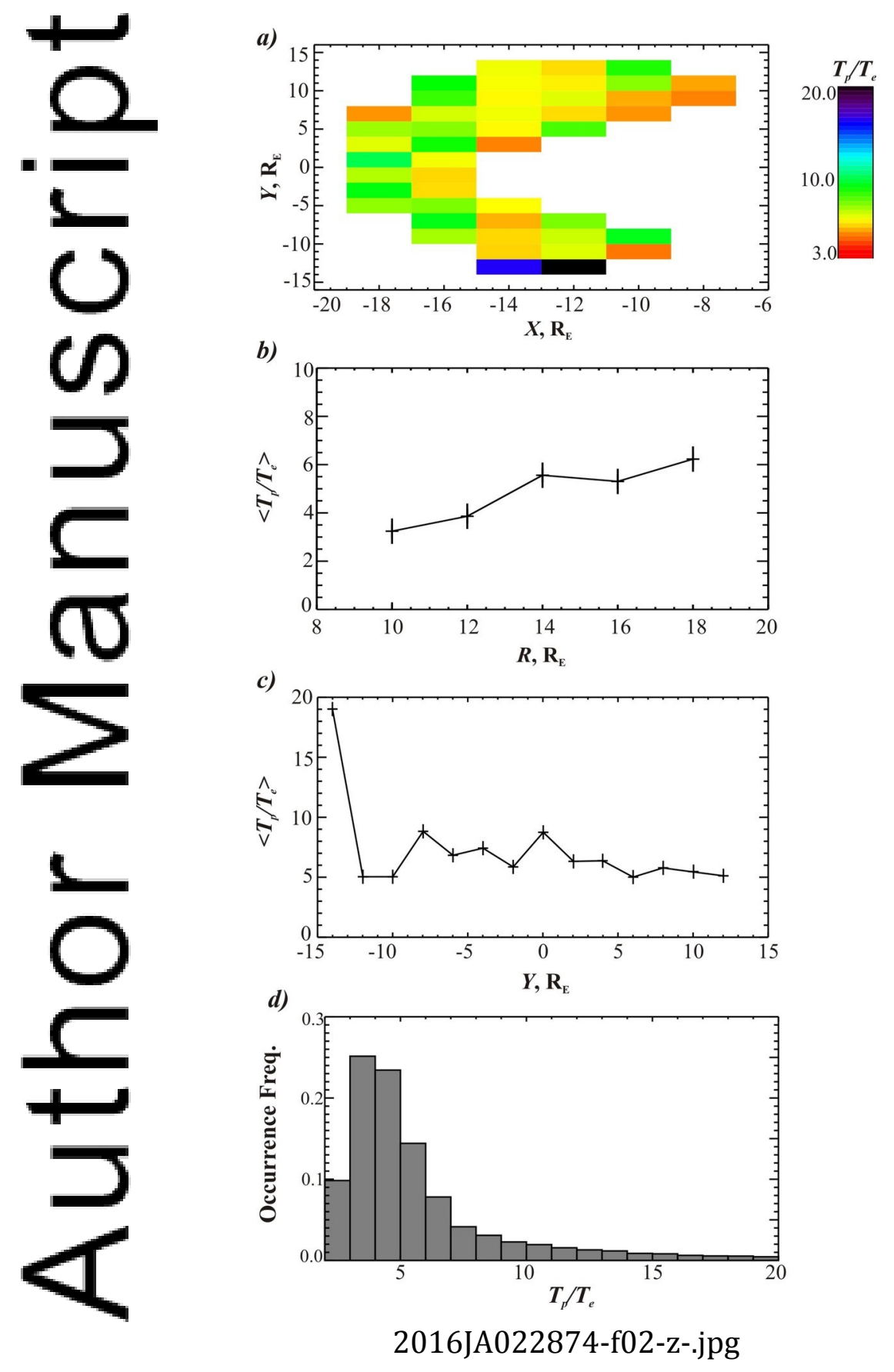

This article is protected by copyright. All rights reserved. 


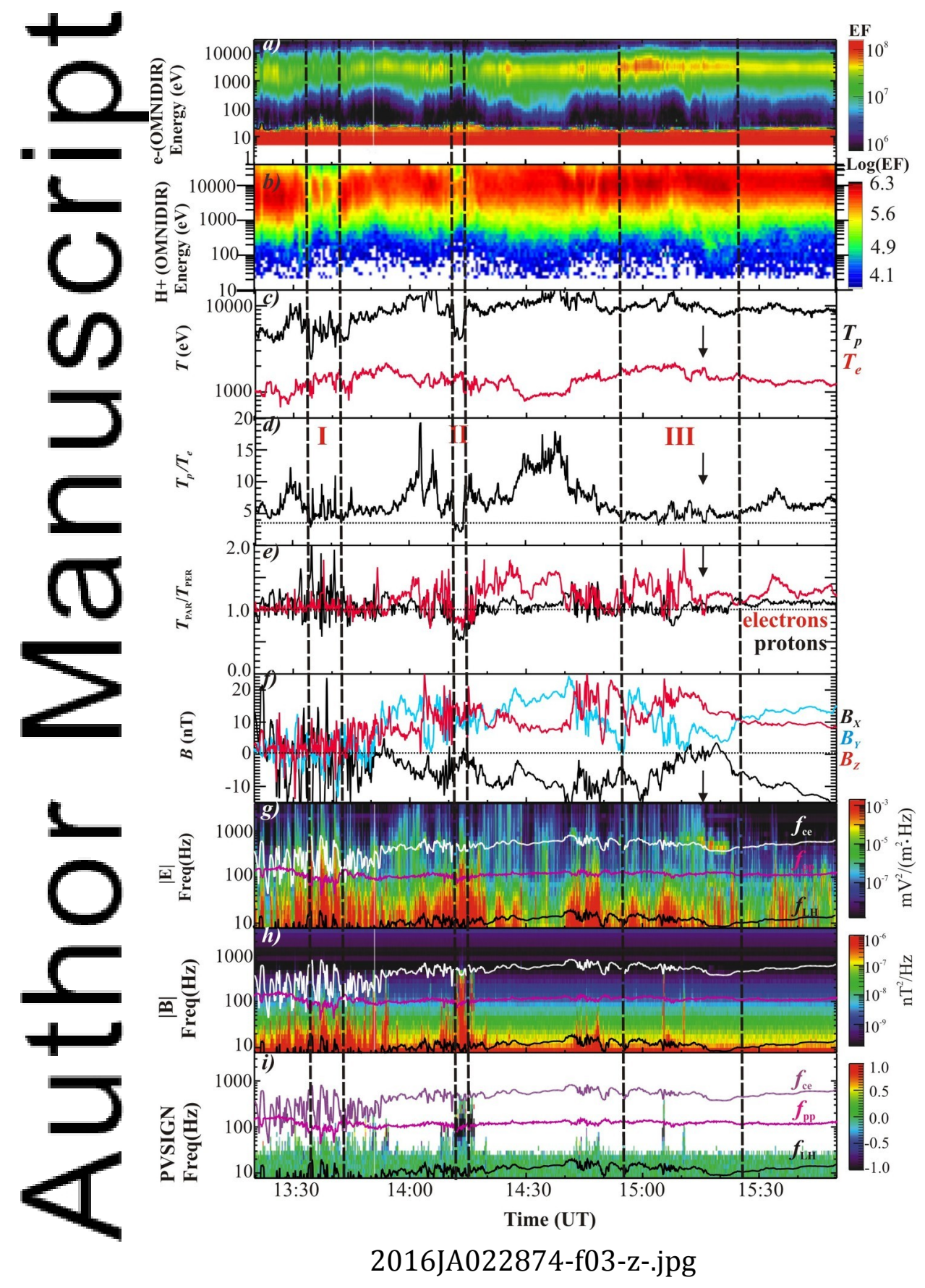

This article is protected by copyright. All rights reserved. 


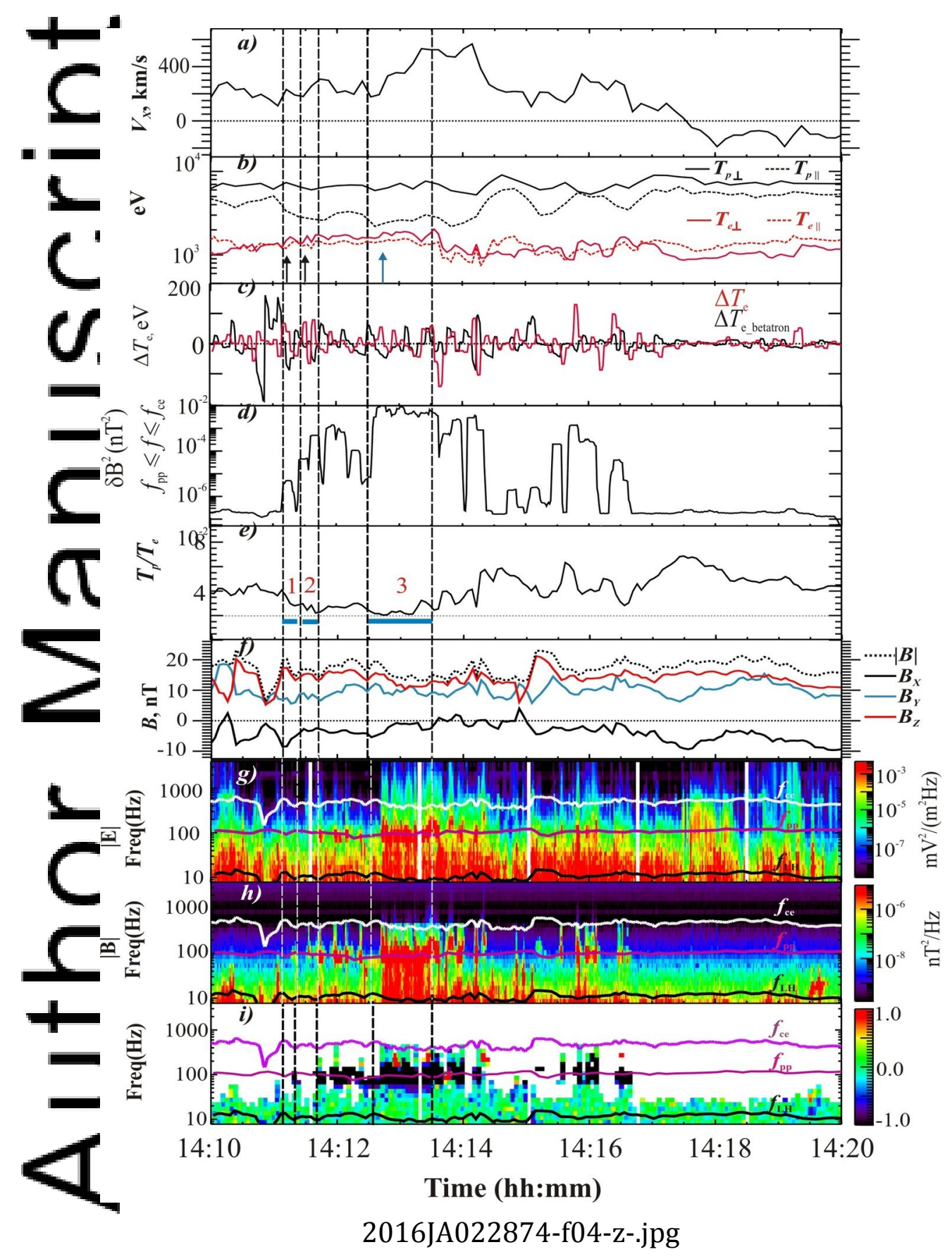

This article is protected by copyright. All rights reserved. 

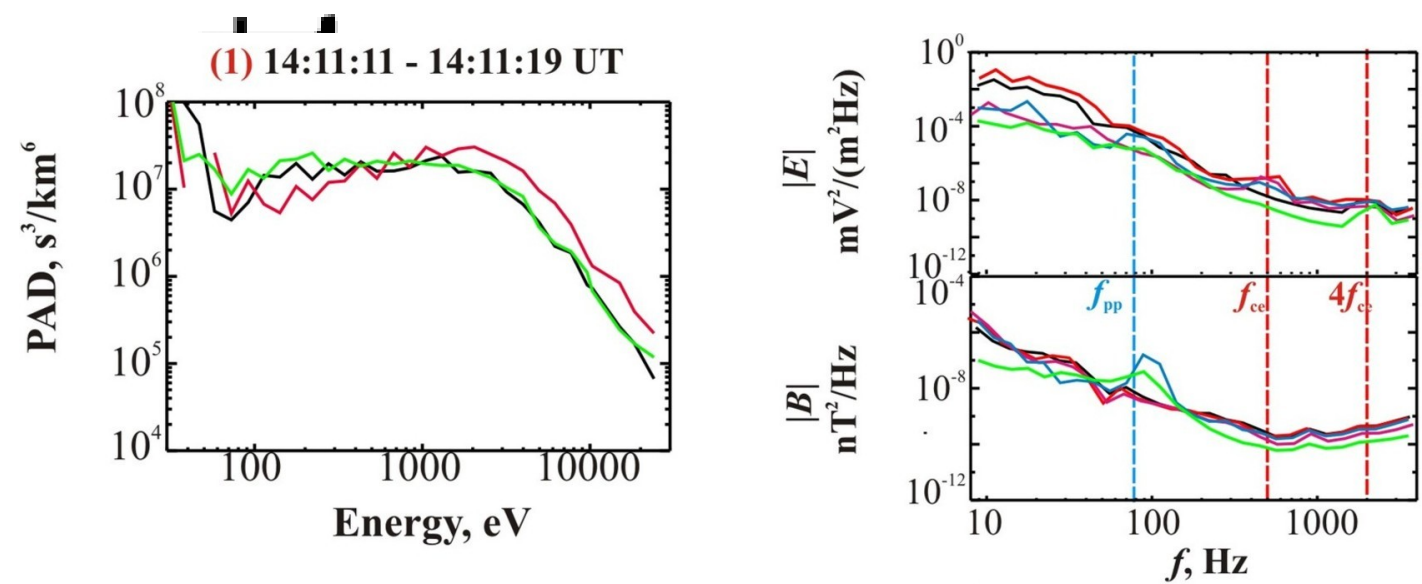

14:11:09 - 14:11:10 UT

$14: 11: 10$ - 14:11:11 UT

$14: 11: 11$ - 14:11:12 UT

14:11:14 - 14:11:19 UT

14:11:19 - 14:11:20 UT
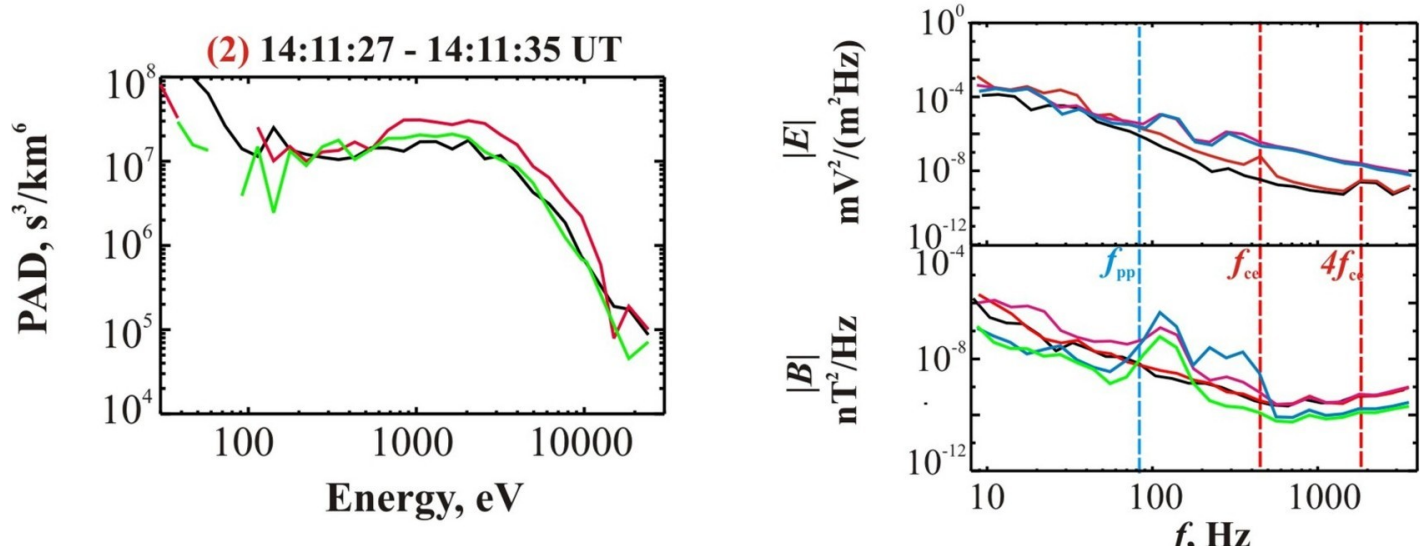

4:11:25 - 14:11:26 UT

$4: 11: 26-14: 11: 27$ UT

$14: 11: 30-14: 11: 31$ UT

$14: 11: 31$ - 14:11:33 UT

14:11:33 - 14:11:34 UT

(3) 14:12:41 - 14:13:37 UT
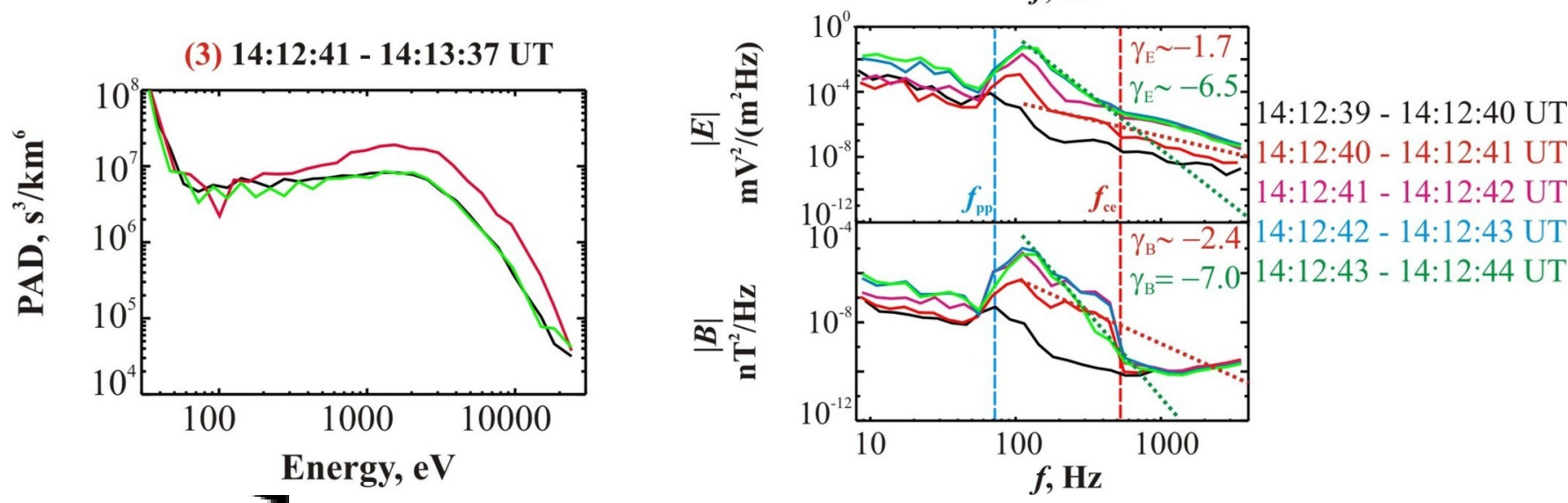

2016JA022874-f05-z-jpg 

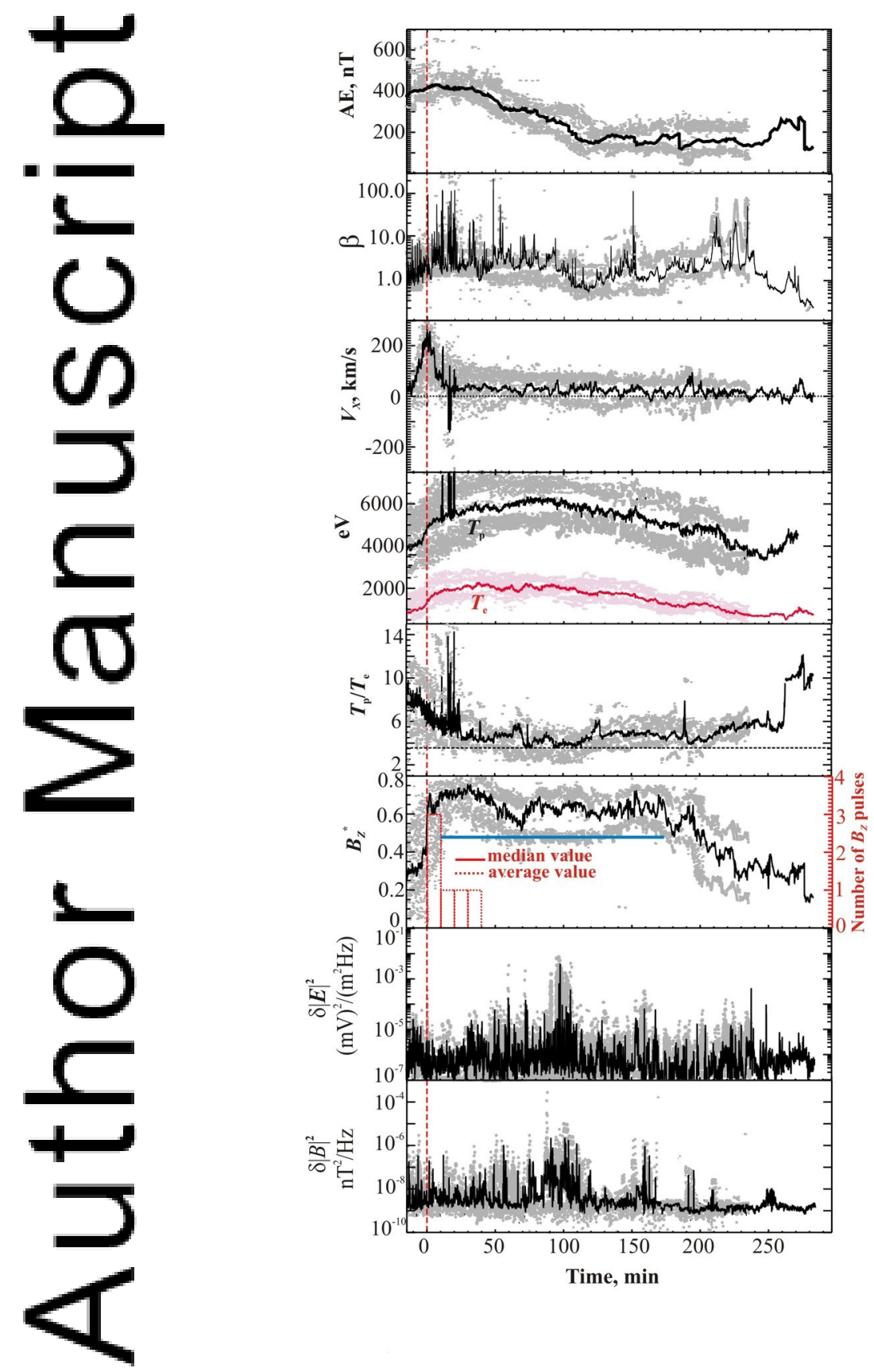

2016JA022874-f06-Z-.jpg 


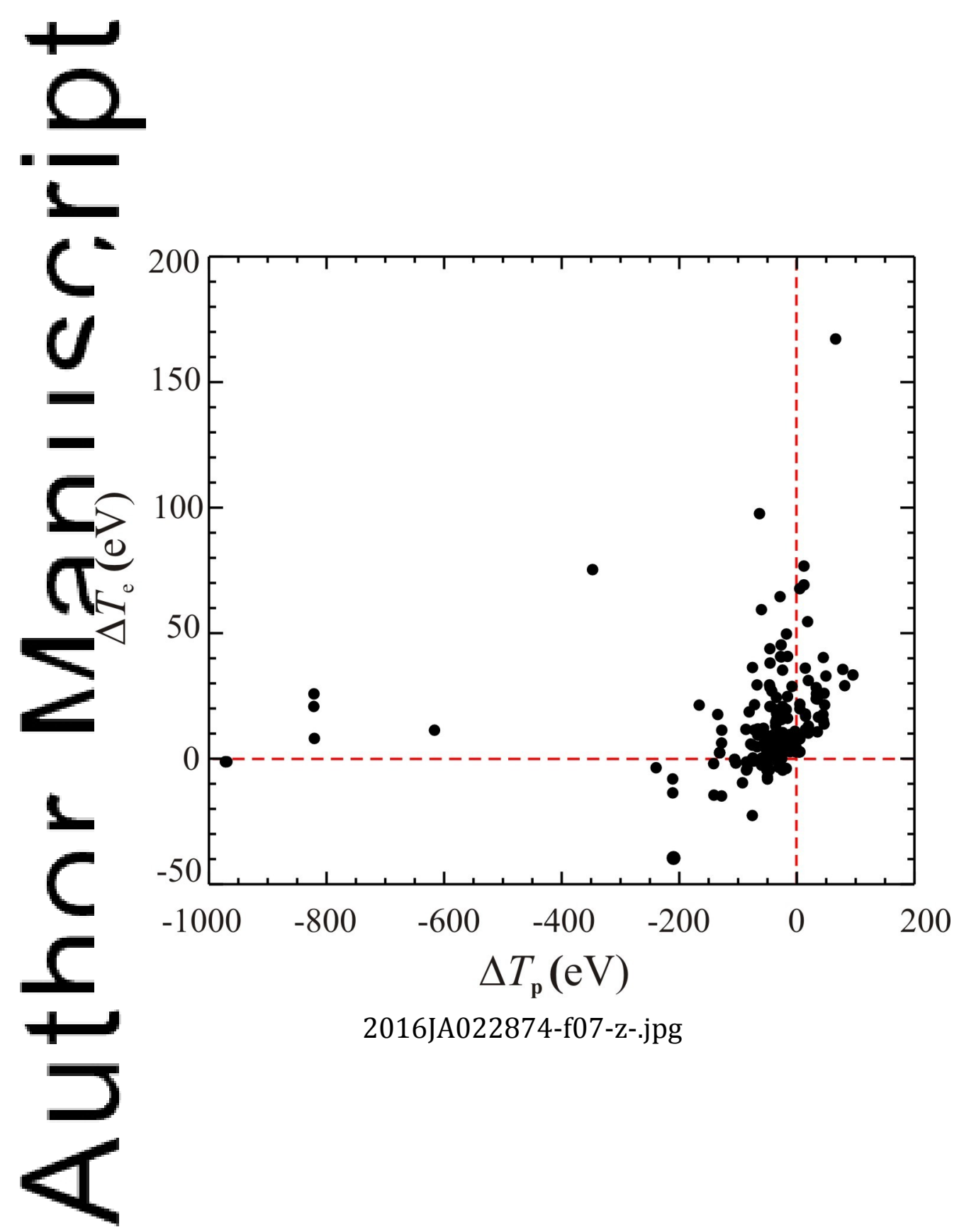

This article is protected by copyright. All rights reserved. 

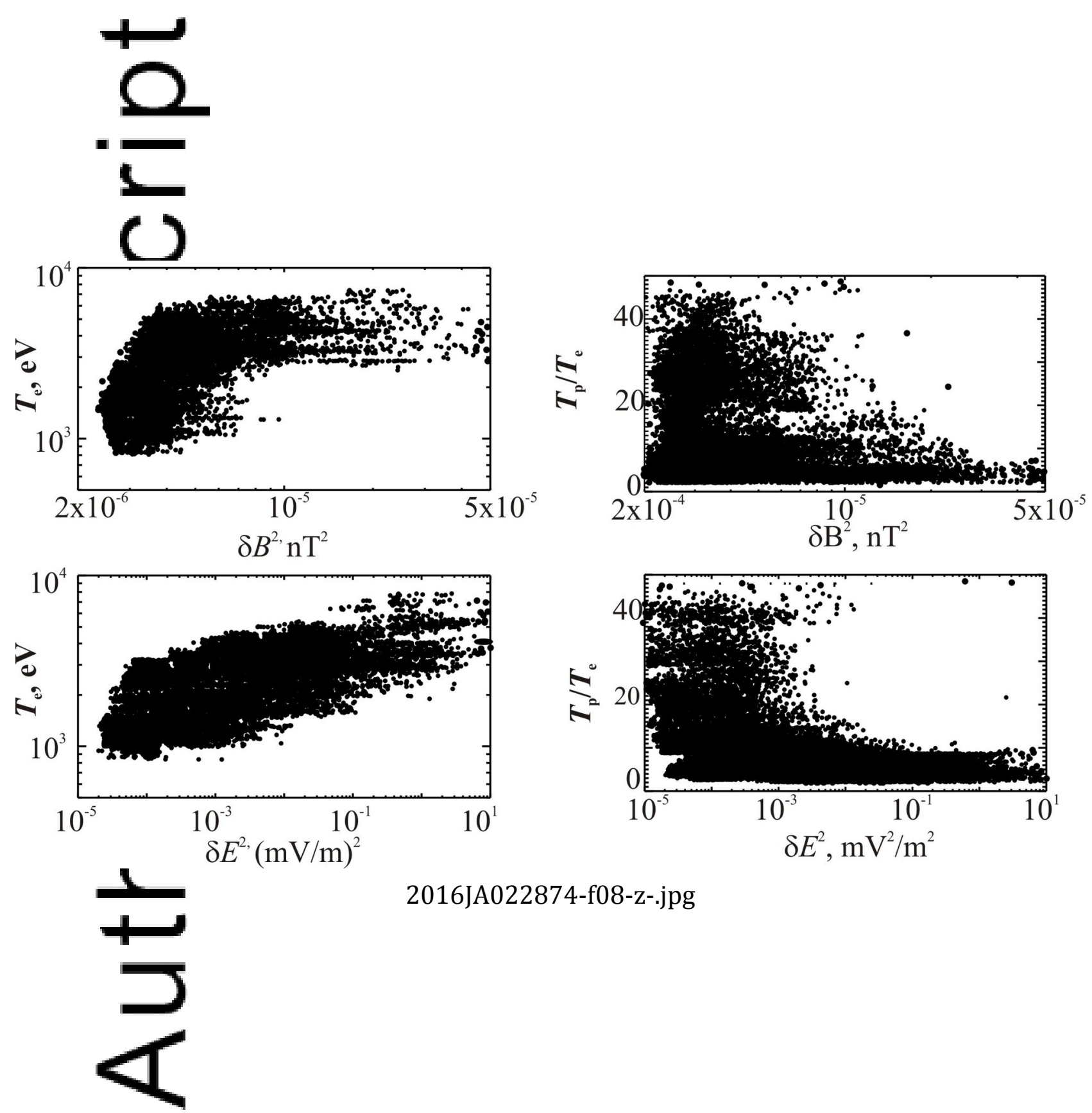

2016JA022874-f08-z-.jpg

This article is protected by copyright. All rights reserved. 